\title{
One-Pot Access to 2-Aryl-3-(arylmethyl)chromones
}

\author{
Meng-Yang Chang*a,b (D) \\ Kuan-Ting Chen ${ }^{a}$ \\ Yu-Lin Tsai ${ }^{\mathrm{a}}$ \\ Han-Yu Chena \\ a Department of Medicinal and Applied Chemistry, \\ Kaohsiung Medical University, Kaohsiung 807, \\ Taiwan \\ ${ }^{b}$ Department of Medical Research, Kaohsiung \\ Medical University Hospital, Kaohsiung 807, \\ Taiwan \\ mychang@kmu.edu.tw
}

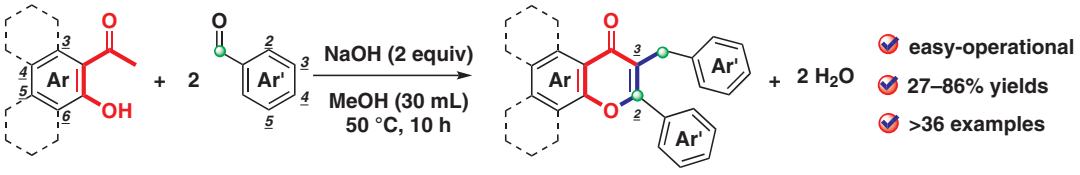

$\mathrm{Ar}=\mathrm{Ph}, 4-\mathrm{FC}_{6} \mathrm{H}_{3}, 4-\mathrm{ClC}_{6} \mathrm{H}_{3}, 4-\mathrm{BrC}_{6} \mathrm{H}_{3}$, 1-naphthyl, 5- $\mathrm{MeOC}_{6} \mathrm{H}_{3}, 4-\mathrm{MeOC}_{6} \mathrm{H}_{3}, 3-\mathrm{MeOC}_{6} \mathrm{H}_{3}, 5-\mathrm{BuOC}_{6} \mathrm{H}_{3}$, 4- $-\mathrm{BuOC}_{6} \mathrm{H}_{3}, 3-\mathrm{BuOC}_{6} \mathrm{H}_{3}, 5-\mathrm{BnOC}_{6} \mathrm{H}_{3}, 4-\mathrm{BnOC}_{6} \mathrm{H}_{3}, 3-\mathrm{BnOC}_{6} \mathrm{H}_{3}, 4,6-\mathrm{Cl}_{2} \mathrm{C}_{6} \mathrm{H}_{2}, 4,6-\mathrm{F}_{2} \mathrm{C}_{6} \mathrm{H}_{2}, 4-\mathrm{MeC}_{6} \mathrm{H}_{3}$, 2-naphthyl, 4- $\mathrm{PhC}_{6} \mathrm{H}_{3}, 4-\left(4-\mathrm{FC}_{6} \mathrm{H}_{4}\right) \mathrm{C}_{6} \mathrm{H}_{3}$, 4-(4- $\left.\mathrm{MeOC}_{6} \mathrm{H}_{4}\right) \mathrm{C}_{6} \mathrm{H}_{3}$, 4-(2-naphthyl) $\mathrm{C}_{6} \mathrm{H}_{3}$, 4-(4- $\left.\mathrm{PhC}_{6} \mathrm{H}_{4}\right) \mathrm{C}_{6} \mathrm{H}_{3}$ $\mathrm{Ar}^{\prime}=$ pyridyl, phenyl, naphthyl, furyl, thienyl, benzofuryl, benzothienyl, quinolinyl
Received: 22.10.2019

Accepted after revision: 14.11.2019

Published online: 28.11 .2019

DOI: 10.1055/s-0039-1690760; Art ID: ss-2019-f0593-op

Abstract Sodium hydroxide controlled intermolecular double aldol condensation of o-hydroxyacetophenones with 2 equivalents of arylaldehydes provides 2-aryl-3-(arylmethyl)chromones (a chimera of flavone and homoisoflavanone) in $\mathrm{MeOH}$ at $50{ }^{\circ} \mathrm{C}$ under mild conditions. The uses of various bases and solvents are investigated for one-pot facile and efficient transformation. A plausible mechanism is proposed.

Key words aldol condensation, o-hydroxyacetophenones, arylaldehydes, chromones, homoisoflavanone, benzopyran-4-one

The structure of a 2-aryl-3-(arylmethyl)chromone, having $\alpha$-arylmethyl and $\beta$-aryl-conjugated groups, is a chimera of flavone and homoisoflavanone. On the basis of the same benzopyran-4-one skeleton (black), both flavone (blue, 2-aryl) and homoisoflavanone (red, 3-arylmethyl) possess an aryl motif and are the key members of benzofused oxacycles (Scheme 1). They are widely present in natural products and serve as multifunctionalized blocks in synthetic fields. ${ }^{1}$ They exhibit diversified bioactive properties, such as antioxidant, anticancer, and antimicrobial activities. $^{2,3}$

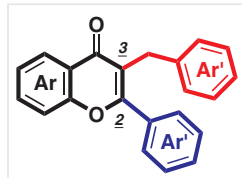

2-aryl-3-(arylmethyl)chromones

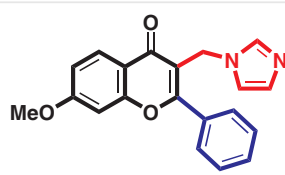

CYP inhibitor (1a)

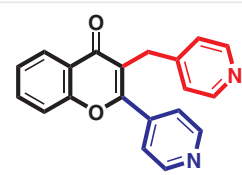

antioxidant agent (1b)
Scheme 1 Structures of 2-aryl-3-(arylmethyl)chromones

Therefore, a number of attempts to synthesize the skeletons of flavone and homoisoflavanone have been reported via various prepared protocols. ${ }^{4,5}$ Comparing two common core skeletons, however, there are fewer studies on the related synthetic works and pharmaceutical properties of 2aryl-3-(arylmethyl)chromones. So far, there are three main groups that have demonstrated the unique biological activities of 2-aryl-3-(arylmethyl)chromones. The Bisi group ${ }^{6 a-c}$ reported that 2-phenyl-3-(imidazolylmethyl)chromones, e.g. 1a, are suitable to serve as flavonoid-based cytochrome P450 (CYP) inhibitors. Lee and co-workers described that 2pyridyl-3-(pyridylmethyl)chromone $\mathbf{1 b}$ is an antioxidant agent. $^{7 \mathrm{a}}$

On the other hand, two attractive examples of the synthesis of 2-aryl-3-(arylmethyl)chromones with the structural hybrid of flavone and homoisoflavanone were described as follows (Scheme 2). They included: (1) Lee and co-workers investigated the ruthenium-catalyzed one-pot $\mathrm{C}-\mathrm{H}$ activation of salicylaldehydes and decarboxylative coupling of alkynoic acids (eq 1), ${ }^{7 a}$ and (2) Liu and co-workers explored the palladium-catalyzed dehydrogenation of 0 acylphenols (eq 2). ${ }^{7 \mathrm{~b}}$ They both showed novel transitionmetal-promoted routes in the formation of 2-aryl-3-(arylmethyl)chromones. In spite of the advancements however, some problems exist, such as complicated catalytic conditions, lack of broad substrate generality, and prefunctionalized fragments. However, an operationally easy, inexpensive, open-vessel, and high-yield route for the preparation of 2-aryl-3-(arylmethyl)chromones with potential bioactive properties had not been reported until recently. As a result of the recent findings, further investigation of efficient synthetic methods for introducing both 2-aryl and 3arylmethyl groups into the core scaffold of benzopyran-4one is still highly desired. Herein, we present a one-pot synthesis of 2-aryl-3-(arylmethyl)chromones $\mathbf{4}$ via alkali-controlled intermolecular double aldol condensation of substituted $\boldsymbol{0}$-hydroxyacetophenones $\mathbf{2}$ with arylaldehydes $\mathbf{3}$ (2 equiv) under warming alcoholic reaction conditions (eq 3 ). 


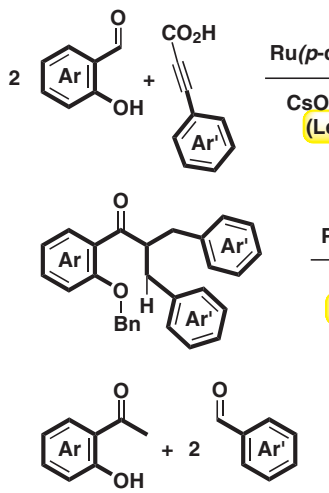

2

3
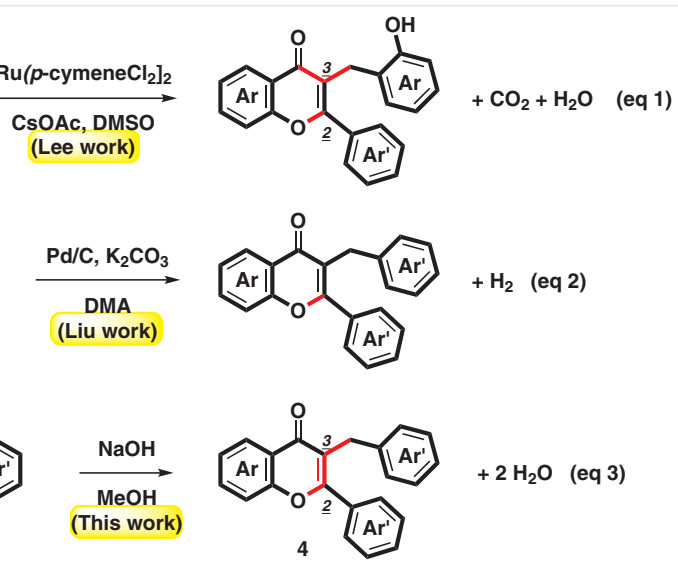

Scheme 2 Synthetic routes toward 2-aryl-3-(arylmethyl)chromones

Continuing our synthetic research on the tandem aldol condensation/Michael addition of 0 -allylbenzaldehydes with different equivalents of substituted acetophenones ( 1 , 2 , and 3 equiv) for the preparation of diversified benzannulated molecules, ${ }^{8}$ a convenient route for synthesizing 2 aryl-3-(arylmethyl)chromones $\mathbf{4}$ was explored next. Using equal equivalents of starting materials, aldol condensation of substituted 0 -hydroxyacetophenones 2 (1 equiv) with arylaldehydes $\mathbf{3}$ ( 1 equiv) provided $\boldsymbol{o}$-hydroxychalcones $\mathbf{5}$ under alkali-mediated traditional conditions. ${ }^{9}$ In particular, when the number of equivalents of arylaldehydes $\mathbf{3}$ was changed from 1 to 2 equiv, 2-aryl-3-(arylmethyl)chromones 4 displaced the expected $\boldsymbol{o}$-hydroxychalcones $\mathbf{5}$ as the domain products in good yields (Scheme 3 ). In fact, to the best of our knowledge, so far no examples of the double aldol condensation of acetophenones $\mathbf{2}$ with arylaldehydes $\mathbf{3}$ have been reported. On the basis of these observations, initially, we utilized 6-bromopicolinaldehyde (3a) as the starting substrate to screen the reaction conditions since the bromo group could introduce a variety of functionalized groups by transition-metal cross-coupling, and the pyridyl ring could perform as a specific pharmacophore. ${ }^{10}$

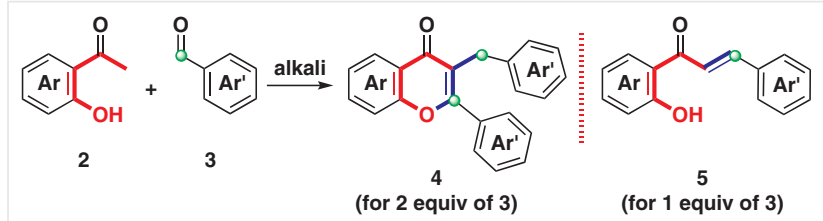

Scheme 3 Alkali-mediated condensation of $\mathbf{2}$ and $\mathbf{3}$

The study commenced with the treatment of model substrates $\mathbf{2 a}(\mathrm{Ar}=\mathrm{Ph}, 1.0 \mathrm{mmol})$ and 6-bromopicolinaldehyde (3a, 2 equiv) in the presence of $\mathrm{NaOH}$ (2 equiv) in $\mathrm{MeOH}(30 \mathrm{~mL})$ at $25^{\circ} \mathrm{C}$ for $10 \mathrm{~h}$ (Table 1 , entry 1$)$. Under these reaction conditions, only trace amounts $(8 \%)$ of $\mathbf{4 a}$ were observed. Then, by controlling the temperature at 25
${ }^{\circ} \mathrm{C}$ and increasing the time to 20,30 , and $40 \mathrm{~h}, \mathbf{4 a}$ was still isolated in low yields (14\%, 16\%, 15\%, entries $2-4)$. Next, elevating the temperature to $50{ }^{\circ} \mathrm{C}$ and controlling the time at $10 \mathrm{~h}$, the yield of $\mathbf{4 a}$ was increased to $86 \%$ (entry 5). After increasing the time from 10 to $20 \mathrm{~h}$, the yield (80\%) of $\mathbf{4 a}$ decreased slightly (entry 6 ). From the results, we found that temperature is a more important factor than time when affecting the formation of $\mathbf{4 a}$. To obtain better yields, we wanted to increase the temperature to reflux $\left(65^{\circ} \mathrm{C}\right)$, but this reflux condition provided a $71 \%$ yield of $\mathbf{4 a}$ (entry 7 ). Furthermore, adjusting the reaction concentrations ( 0.033 $\rightarrow 0.10,0.02 \mathrm{M}$ ) was examined. We found that concentrated solution $(0.1 \mathrm{M})$ provided $\mathbf{4 a}$ in a $25 \%$ yield along with major complex unknown products (entry 8 ). On the other hand, the diluted solution $(0.02 \mathrm{M})$ could maintain the yield at $79 \%$ (entry 9). From the phenomenon, we understood that the factor of reaction concentration also affected the yield. Three alkalis with different basicity levels were examined next. Entries 10 and 11 show that no better yields were detected using $\mathrm{LiOH}$ and $\mathrm{KOH}$ (65\% and 78\%), respectively. Using $\mathrm{K}_{2} \mathrm{CO}_{3}$, the desired $\mathbf{4 a}$ could not be generated (entry 12). By controlling the combination of $50{ }^{\circ} \mathrm{C}$ and 10 $\mathrm{h}$, the solvent was adjusted from $\mathrm{MeOH}$ to $\mathrm{EtOH}$, however only a $58 \%$ yield of 4 a was formed (entry 13 ). After elevating the temperature from $50{ }^{\circ} \mathrm{C}$ to $70{ }^{\circ} \mathrm{C}$, the yield was still maintained at $68 \%$ (entry 14). Entries 15 and 16 show that $i \mathrm{PrOH}$ and $t \mathrm{BuOH}$ did not give better yields (37\% and $12 \%$ ) than $\mathrm{MeOH}(86 \%)$. By using $\mathrm{H}_{2} \mathrm{O}$ as the solvent, no reaction was observed due to the poor solubility (entry 17). In entries 18 and 19, attempts to increase the number of equivalents ( $2 \rightarrow 3$ or 4 ) failed to afford higher yields ( $82 \%$ or $75 \%$ ). Then, 1 equivalent of $\mathrm{NaOH}$ was tested, and only a $45 \%$ yield of $\mathbf{4 a}$ was detected (entry 20). From our observations, we concluded that $\mathrm{NaOH}$ was the optimal alkali to yield $\mathbf{4 a}$ under warming methanolic reaction conditions via one-pot facile intermolecular double condensation of $\mathbf{2 a}$ and $\mathbf{3 a}$. 
Table 1 Reaction Conditions ${ }^{\mathrm{a}}$

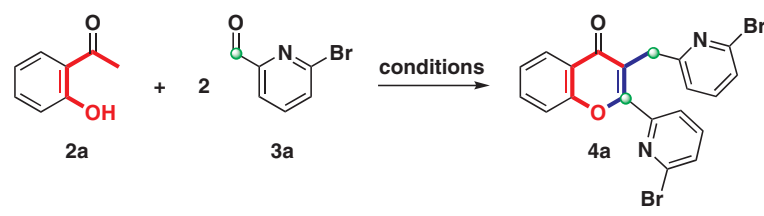

\begin{tabular}{|c|c|c|c|c|c|}
\hline Entry & Alkali & Solvent (mL) & Temp $\left({ }^{\circ} \mathrm{C}\right)$ & Time (h) & Yield $^{\mathrm{b}}(\%)$ \\
\hline 1 & $\mathrm{NaOH}$ & $\mathrm{MeOH}(30)$ & 25 & 10 & 8 \\
\hline 2 & $\mathrm{NaOH}$ & $\mathrm{MeOH}(30)$ & 25 & 20 & 14 \\
\hline 3 & $\mathrm{NaOH}$ & $\mathrm{MeOH}(30)$ & 25 & 30 & 16 \\
\hline 4 & $\mathrm{NaOH}$ & $\mathrm{MeOH}(30)$ & 25 & 40 & 15 \\
\hline 5 & $\mathrm{NaOH}$ & $\mathrm{MeOH}(30)$ & 50 & 10 & 86 \\
\hline 6 & $\mathrm{NaOH}$ & $\mathrm{MeOH}(30)$ & 50 & 20 & 80 \\
\hline 7 & $\mathrm{NaOH}$ & $\mathrm{MeOH}(30)$ & reflux (65) & 10 & 71 \\
\hline 8 & $\mathrm{NaOH}$ & $\mathrm{MeOH}(10)$ & 50 & 10 & $25^{c}$ \\
\hline 9 & $\mathrm{NaOH}$ & $\mathrm{MeOH}(50)$ & 50 & 10 & 79 \\
\hline 10 & $\mathrm{LiOH}$ & $\mathrm{MeOH}(30)$ & 50 & 10 & 65 \\
\hline 11 & $\mathrm{KOH}$ & $\mathrm{MeOH}(30)$ & 50 & 10 & 78 \\
\hline 12 & $\mathrm{~K}_{2} \mathrm{CO}_{3}$ & $\mathrm{MeOH}(30)$ & 50 & 10 & $-{ }^{d}$ \\
\hline 13 & $\mathrm{NaOH}$ & $\mathrm{EtOH}(30)$ & 50 & 10 & 71 \\
\hline 14 & $\mathrm{NaOH}$ & EtOH (30) & 70 & 10 & 68 \\
\hline 15 & $\mathrm{NaOH}$ & iPrOH (30) & 50 & 10 & 37 \\
\hline 16 & $\mathrm{NaOH}$ & $\mathrm{tBuOH}(30)$ & 50 & 10 & 12 \\
\hline 17 & $\mathrm{NaOH}$ & $\mathrm{H}_{2} \mathrm{O}(30)$ & 50 & 10 & $--^{d}$ \\
\hline 18 & $\mathrm{NaOH}^{\mathrm{e}}$ & $\mathrm{MeOH}(30)$ & 50 & 10 & 82 \\
\hline 19 & $\mathrm{NaOH}^{f}$ & $\mathrm{MeOH}(30)$ & 50 & 10 & 75 \\
\hline 20 & $\mathrm{NaOH}^{9}$ & $\mathrm{MeOH}(30)$ & 50 & 10 & 45 \\
\hline
\end{tabular}

a Reaction conditions: $2 \mathbf{2 a}(1.0 \mathrm{mmol}), 3 \mathbf{3}$ ( 2 equiv), alkali ( 2 equiv).

b Isolated yields of 4 a.

' Unknown mixture was isolated.

${ }^{\mathrm{d}}$ No product detected.

e 3 equiv.

f 4 equiv.

g 1 equiv.

On the basis of our experimental results, a plausible mechanism for the formation of $\mathbf{4 a}$ is illustrated in Scheme 4. Initially, $\mathrm{NaOH}$ (2 equiv) mediated deprotonation of $\mathbf{2 a}$ provided a dianion intermediate. Following, in situ formed $\alpha$-carbanion which attacked the carbonyl group of $\mathbf{3 a}$ to lead to $\mathbf{A}$. Then, an intramolecular proton exchange of $\mathbf{A}$ with two oxyanions yielded $\mathbf{B}$. By the involvement of another 3a, B was converted into $\mathbf{C}$ via a second intermolecular aldol reaction. Subsequently, D having the delocalized $\alpha$ carbanion, was generated again by the repeated intramolecular proton exchange of $\mathbf{C}$. After releasing the hydroxide ion on D, E with a conjugated enone moiety was formed. Furthermore, the oxyanion promoted the oxy-Michael reaction to the enone moiety followed by elimination of another hydroxide ion, and afforded (E)- or (Z)-homoisoflavone skeleton $\mathbf{F}$ via an intramolecular ring-closure process. Finally, 1,3-hydride migration on $\mathbf{F}$ from trisubstituted exo-olefin to tetrasubstituted endo-olefin furnished the construction of 4a. On the other pathway, removal of the hydroxyl group of B provided $\mathbf{G}$ with a chalcone group. By intramolecular oxaMichael annulation of $\mathbf{G}$ followed by intermolecular aldol

Table 2 Synthesis of $\mathbf{4 a - 4 a b ^ { a }}$

\begin{tabular}{llll} 
& & & \\
\hline
\end{tabular}

a Reaction conditions: $\mathbf{2 a}-\mathbf{2 w}$ (1.0 mmol) 3a-3f ( 2 equiv), $\mathrm{NaOH}(80 \mathrm{mg}, 2$ equiv), $\mathrm{MeOH}(30 \mathrm{~mL}), 50^{\circ} \mathrm{C}, 10 \mathrm{~h}$.

${ }^{\mathrm{b}} \mathrm{Ar}$ is the substituted arene ring in 2-formylaren-1-ol (numbering shown).

' $\mathrm{Ar}$ ' is the substituted pyridine ring in 2-formylpyridine (numbering shown).

dIsolated yields.

e Substrate was 1-hydroxynaphthalene-2-carbaldehyde.

${ }^{f}$ Substrate was 2-hydroxynaphthalene-1-carbaldehyde. 

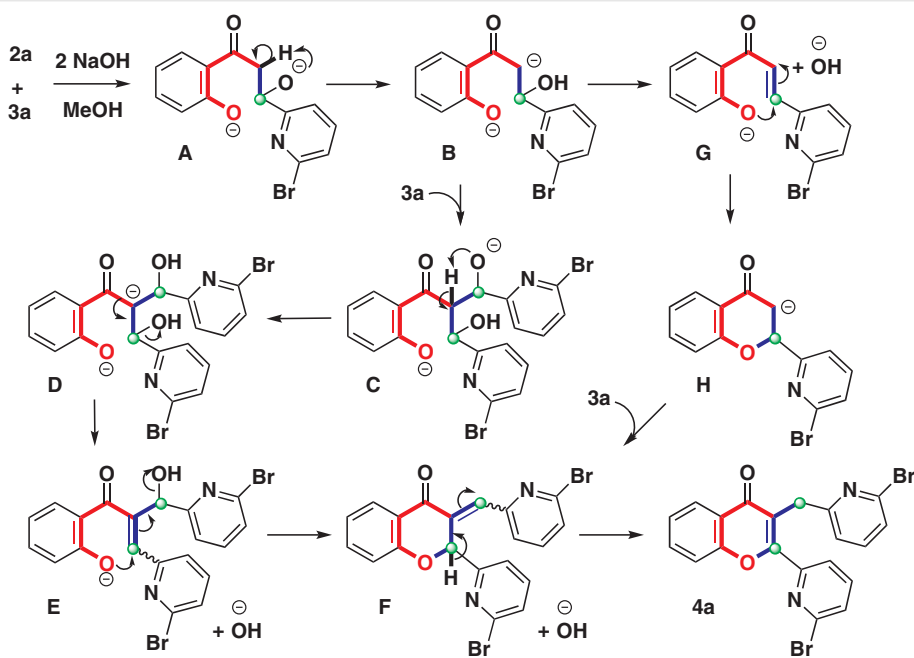

Scheme 4 Plausible mechanism

condensation of the resulting $\mathbf{H}$ with $\mathbf{3 a}, \mathbf{F}$ was achieved. Based on the above-mentioned procedure, $\mathbf{4 a}$ was constructed. From the possible mechanism, we found that $\mathrm{NaOH}$ efficiently organized the continuous sequence of intermolecular aldol reactions and intramolecular ringclosures.

To study the scope and limitations of this approach, substituted o-hydroxyacetophenones $\mathbf{2 a - 2 w}$ and 2formylpyridines $\mathbf{3 a} \mathbf{a}-\mathbf{3 f}$ were reacted with $\mathrm{NaOH}$ ( 2 equiv) to afford diversified 2-aryl-3-(arylmethyl)chromones 4a-4ab under warming methanol $\left(50^{\circ} \mathrm{C} / 10 \mathrm{~h}\right)$ conditions, as shown in Table 2. With optimal conditions established (Table 1, entry 5) and a plausible mechanism proposed (Scheme 4), we found that this route allowed direct double aldol condensation in moderate to good yields (64-86\%). Among entries 128, efficient formation of $\mathbf{4 a - 4 a b}$ showed that the two aryl substituents (2, $\mathrm{Ar}$ and $\left.3, \mathrm{Ar}^{\prime}\right)$ did not affect the yields. The structures of $\mathbf{4 a - 4 d}, \mathbf{4 g}, \mathbf{4 t}$, and $\mathbf{4 z}$ were determined by single-crystal X-ray crystallography. ${ }^{11}$ For the electronic nature of aryl substituents ( $\mathrm{Ar}$ ) of 2, not only haloaryl (bromo, chloro, dichloro) groups but also electron-neutral (methyl), electron-withdrawing (fluoro, difluoro) and electrondonating (methoxy, butoxy, benzyloxy) groups were appropriate. For the pyridyl substituents $\left(\mathrm{Ar}^{\prime}\right)$ of 3, 6-bromo, 5fluoro, 6-methyl, 6-chloro, and 6-methoxy groups were well-tolerated. Based on the results, after replacing the pyridyl group of $\mathbf{3 a - 3 f}$ with benzaldehyde (3g) and 2-naphthaldehyde (3h), synthesis of 4ac-4ad was examined next (Scheme 5). However, the desired 4 ac and 4ad were formed in $43 \%$ and $36 \%$ yields, respectively. Next, when the monocyclic 2-formyl-heteroarenes were changed from 2formylpyridines $\mathbf{3 a}-\mathbf{3 f}$ to 1 -allyl-2-formyl-1 $H$-pyrrole (3i), 2-formylfuran (3j), 2-formylthiophene (3k), and 3- formylpyridine (31), 4af and 4ag were produced with moderate results (58\% and 60\% yields); surprisingly, the desired 4ae could be not isolated. The structure of $\mathbf{4 a g}$ was determined by single-crystal X-ray crystallography. ${ }^{11}$ In particular, 4 ah was provided in only a $27 \%$ yield. Then, changing to bicyclic 2-formylbenzofuran (3m), 2-formylbenzothiophene (3n), and 3-formyl-1-methyl-1H-indole (3o), 4ai and 4aj were obtained in 64\%, and 63\% yields, respectively. However, the desired 4ak could be not isolated, and only $15 \%$ yield of chalcone 4ak-1 was obtained. Furthermore, 2formylquinoline (3p) produced 63\% yield of 4 al.

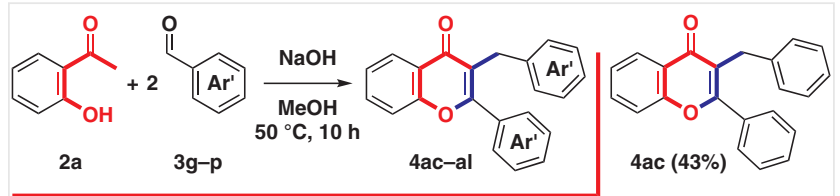<smiles>O=C1C2C=CC=CC2OC(c2ccc3ccccc3c2)C1Cc1ccc2ccccc2c1</smiles>
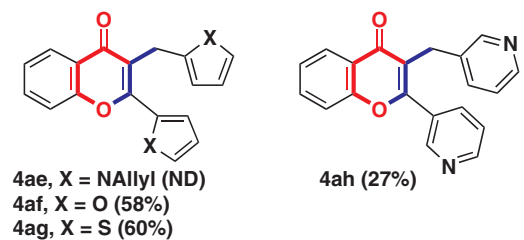<smiles>[X][X]1c(CC2Oc3ccccc3C(=O)C2Cc2ccccc2[X])cc2ccccc12</smiles>

$4 a \mathrm{i}, \mathrm{X}=\mathrm{O}(64 \%)$ $4 \mathrm{aj}, \mathrm{X}=\mathrm{S}(63 \%)$
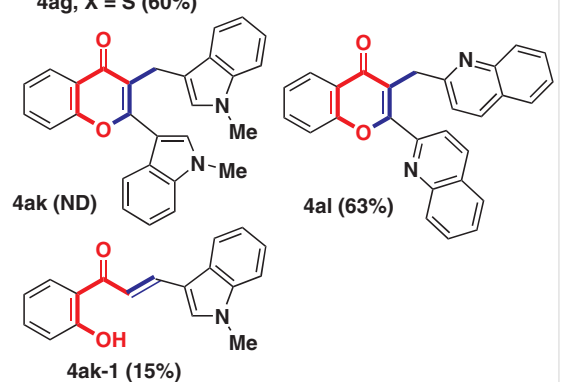

Scheme 5 Synthesis of $\mathbf{4 a c}-\mathbf{4 a l}$ and $\mathbf{4 a k}-\mathbf{1} ; \mathrm{ND}=$ not detected 

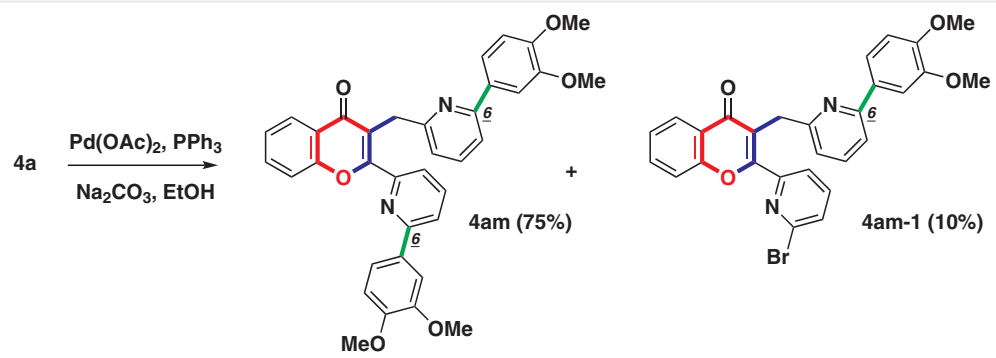

Scheme 6 Suzuki-Miyaura coupling of 4 a

From the results, we understand that hetero-monocyclic 2-azaarenes could produce better yields than benzaldehyde and naphthaldehyde, especially with substituted 2formylpyridines. The putative explanations for the differences are that the aggregated intermediate adopts a bidentate coordination of the sodium ion by the nitrogen lonepair of 2-formylpyridine and the alkoxy anion such that this resulting complexation would necessitate the intermediate to coordinate with the second equivalent of 2 -formylpyridine via the aldol reaction process. ${ }^{12}$

To elongate the side arm on the pyridyl ring of 4a, SuzukiMiyaura coupling of 4a with 3,4-dimethoxyphenylboronic acid provided 4am in a $75 \%$ yield along with a $10 \%$ yield of 4am-1, as shown in Scheme 6. With the results, we envisioned that different aryl substituents could be installed into the 6-position of pyridine ring on $\mathbf{4 a}$ to afford diversified aryl-pyridyl-conjugated homoisoflavanones.

On the other hand, a macrocyclic isoflavanone was examined (Scheme 7). Under the above conditions, a one-pot $\mathrm{NaOH}$-controlled reaction of $\mathbf{2 a}$ and $\mathbf{3 q}$ with the 0 -allyl side chain provided flavone $4 a n$ in a $40 \%$ yield. In particular, no desired homoisoflavanone was detected. The possible reason should be that the 0 -allyl group on 3q exhibited a bulkier steric hindrance such that the second aldol condensation could not be generated. For the formation of 4an, we thought that in situ generated I could trap molecular oxygen (from air) to lead II. Following, by the peroxyanionmediated intramolecular deprotonation of II, 4an was accomplished.

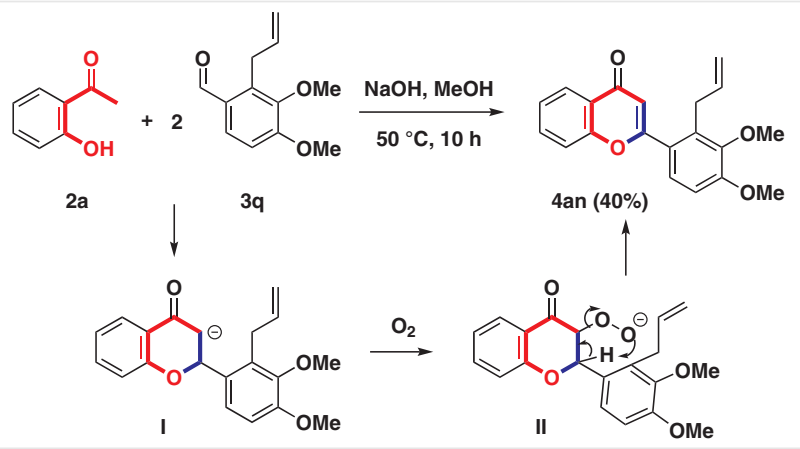

Scheme 7 Synthesis of 4 an
In summary, we have developed an $\mathrm{NaOH}$-controlled synthesis of 2-aryl-3-(arylmethyl)chromones via intermolecular double aldol condensation of $o$-hydroxyacetophenones with 2 equivalents of an arylaldehyde under warming $\mathrm{MeOH}$ reaction conditions in moderate to good yields. The process provides a cascade pathway of $\mathrm{C}-\mathrm{C}$ and then, $\mathrm{C}-\mathrm{O}$ bond formations. The uses of various bases and solvents are investigated for one-pot facile and efficient transformation. Related plausible mechanisms have been proposed. The structures of the key products were confirmed by X-ray crystallography. Further investigations regarding the synthetic applications of $o$-hydroxyacetophenones will be conducted and published in due course.

All reagents and solvents were obtained from commercial sources and used without further purification. Reactions were routinely carried out under an atmosphere of dry air with magnetic stirring. The heating mantle is used to provide a stable heat source. Products in organic solvents were dried with anhydrous $\mathrm{MgSO}_{4}$ before concentration in vacuo. Melting points were determined with a SMP3 melting apparatus. ${ }^{1} \mathrm{H}$ and ${ }^{13} \mathrm{C}$ NMR spectra were recorded on a Varian INOVA400 spectrometer operating at 400 and at $100 \mathrm{MHz}$, respectively. HRMS were measured with a mass spectrometer Finnigan/Thermo Quest MAT 95XL. X-ray crystal structures were obtained with an Enraf-Nonius FR-590 diffractometer (CAD4, Kappa CCD).

For the starting compounds $\mathbf{2} \mathbf{a}-\mathbf{2} \mathbf{w}$ and $\mathbf{3 a} \mathbf{a} \mathbf{3 r}$, these reagents were obtained from commercial sources and used without further purification.

2-Aryl-3-(arylmethyl)chromen-4-ones 4a-4ad, 4af-4aj, 4ak-1, and 4al; General Procedure

$\mathrm{NaOH}(80 \mathrm{mg}, 2.0 \mathrm{mmol})$ was added to a solution of $\mathbf{2 a}-\mathbf{2} \mathbf{w}(1.0$ $\mathrm{mmol}$ ) in $\mathrm{MeOH}(30 \mathrm{~mL})$ at $25^{\circ} \mathrm{C}$. The mixture was stirred at $25^{\circ} \mathrm{C}$ for $10 \mathrm{~min}$. Arylaldehyde $3 \mathbf{a}-\mathbf{3 p}(2.0 \mathrm{mmol})$ was added to the mixture at $25^{\circ} \mathrm{C}$ and it was stirred at $50{ }^{\circ} \mathrm{C}$ for $10 \mathrm{~h}$. The mixture was cooled to $25^{\circ} \mathrm{C}$ and the solvent was concentrated. The residue was diluted with water $(10 \mathrm{~mL})$ and the mixture was extracted with $\mathrm{CH}_{2} \mathrm{Cl}_{2}(3 \times 20 \mathrm{~mL})$. The combined organic layers were washed with brine $(2 \times 10 \mathrm{~mL})$, dried $\left(\mathrm{MgSO}_{4}\right)$, filtered and evaporated to afford crude product under reduced pressure. The remaining mixture was separated by column chromatography (silica gel, hexanes/EtOAc 20:1-3:1) affording the title compounds. 


\section{2-(6-Bromopyridin-2-yl)-3-(6-bromopyridin-2-ylmethyl)-} chromen-4-one (4a)

Colorless solid; yield: $404 \mathrm{mg}$ (86\%); mp $179-180^{\circ} \mathrm{C}$ (hexanes/EtOAc). ${ }^{1} \mathrm{H} \mathrm{NMR}\left(400 \mathrm{MHz}, \mathrm{CDCl}_{3}\right): \delta=8.21(\mathrm{dd}, J=8.0,1.6 \mathrm{~Hz}, 1 \mathrm{H}), 8.06(\mathrm{dd}$, $J=8.0,0.8 \mathrm{~Hz}, 1 \mathrm{H}), 7.72(\mathrm{t}, J=8.0 \mathrm{~Hz}, 1 \mathrm{H}), 7.69(\mathrm{dd}, J=8.8,2.0 \mathrm{~Hz}, 1$ $\mathrm{H}), 7.56(\mathrm{dd}, J=8.0,0.8 \mathrm{~Hz}, 1 \mathrm{H}), 7.51(\mathrm{dd}, J=8.8,0.8 \mathrm{~Hz}, 1 \mathrm{H}), 7.42(\mathrm{dt}$, $J=8.0,1.2 \mathrm{~Hz}, 1 \mathrm{H}), 7.37(\mathrm{~d}, J=7.6 \mathrm{~Hz}, 1 \mathrm{H}), 7.23(\mathrm{dd}, J=8.0,0.4 \mathrm{~Hz}, 1$ $\mathrm{H}), 7.21(\mathrm{dd}, J=8.0,0.4 \mathrm{~Hz}, 1 \mathrm{H}), 4.32(\mathrm{~s}, 2 \mathrm{H})$.

${ }^{13} \mathrm{C}\left\{{ }^{1} \mathrm{H}\right\}$ NMR $\left(100 \mathrm{MHz}, \mathrm{CDCl}_{3}\right): \delta=178.2,161.2,158.4,155.8,152.1$, $141.4,141.0,138.9,138.8,134.0,129.3,126.0,125.2,125.2,123.2$, $122.8,121.8,120.4,118.1,32.9$.

HRMS (ESI-TOF): $m / z[\mathrm{M}+\mathrm{H}]^{+}$calcd for $\mathrm{C}_{20} \mathrm{H}_{13} \mathrm{Br}_{2} \mathrm{~N}_{2} \mathrm{O}_{2}: 470.9344$; found: 470.9338 .

Single-crystal X-ray diagram: crystal of compound $\mathbf{4 a}$ was grown by slow diffusion of EtOAc into a solution of compound $4 \mathbf{a}$ in $\mathrm{CH}_{2} \mathrm{Cl}_{2}$ to yield colorless prisms. The compound crystallizes in the orthorhombic crystal system, space group Pbca, $a=11.6446$ (3) $\AA, b=14.7976$ (4) $\AA ̊, c=19.6780(6) \AA ̊, V=3390.76(16) \AA^{3}, Z=8, d_{\text {calcd }}=1.850 \mathrm{~g} / \mathrm{cm}^{3}$, $F(000)=1856,2 \theta$ range $2.070-26.456^{\circ}, R$ indices (all data) $R 1=$ $0.0233, w R 2=0.0499$.

\section{2-(6-Bromopyridin-2-yl)-3-(6-bromopyridin-2-ylmethyl)-6-fluo- rochromen-4-one $(4 \mathrm{~b})$}

Colorless solid; yield: $420 \mathrm{mg}$ (86\%); mp $190-191{ }^{\circ} \mathrm{C}$ (hexanes/EtOAc). ${ }^{1} \mathrm{H} \mathrm{NMR}\left(400 \mathrm{MHz}, \mathrm{CDCl}_{3}\right): \delta=8.05(\mathrm{~d}, J=7.2 \mathrm{~Hz}, 1 \mathrm{H}), 7.81(\mathrm{dd}, J=8.0$, $3.2 \mathrm{~Hz}, 1 \mathrm{H}), 7.71(\mathrm{t}, J=8.0 \mathrm{~Hz}, 1 \mathrm{H}), 7.55(\mathrm{dd}, J=8.0,0.8 \mathrm{~Hz}, 1 \mathrm{H}), 7.51$ (dd, $J=9.2,4.0 \mathrm{~Hz}, 1 \mathrm{H}), 7.43-7.36(\mathrm{~m}, 2 \mathrm{H}), 7.23(\mathrm{~d}, J=7.6 \mathrm{~Hz}, 1 \mathrm{H})$, $7.21(\mathrm{~d}, J=8.0 \mathrm{~Hz}, 1 \mathrm{H}), 4.31(\mathrm{~s}, 2 \mathrm{H})$.

${ }^{13} \mathrm{C}\left\{{ }^{1} \mathrm{H}\right\}$ NMR $\left(100 \mathrm{MHz}, \mathrm{CDCl}_{3}\right): \delta=177.5(\mathrm{~d}, J=2.3 \mathrm{~Hz}), 160.9,159.5$ (d, $J=245.6 \mathrm{~Hz}), 158.2,152.0$ (d, $J=1.5 \mathrm{~Hz}), 151.8,141.4,141.0,139.0$, 138.5, 129.4, 125.3, $123.8(\mathrm{~d}, J=8.6 \mathrm{~Hz}), 123.3,122.2(\mathrm{~d}, J=25.7 \mathrm{~Hz})$, 121.9, 120.3 (d, $J=7.6 \mathrm{~Hz}), 119.8,110.7$ (d, $J=23.5 \mathrm{~Hz}), 32.7$.

HRMS (ESI-TOF): $m / z$ [M + H] $]^{+}$calcd for $\mathrm{C}_{20} \mathrm{H}_{12} \mathrm{Br}_{2} \mathrm{FN}_{2} \mathrm{O}_{2}$ : 488.9250; found: 488.9244 .

Single-crystal X-ray diagram: crystal of compound $\mathbf{4 b}$ was grown by slow diffusion of EtOAc into a solution of compound $\mathbf{4 b}$ in $\mathrm{CH}_{2} \mathrm{Cl}_{2}$ to yield colorless prisms. The compound crystallizes in the monoclinic crystal system, space group $P 2_{1} / c, a=14.5142(8) \AA, b=19.8390(9) \AA$, $c=12.2505(6) \AA, V=3521.0(3) \AA^{3}, Z=4, d_{\text {calcd }}=1.849 \mathrm{~g} / \mathrm{cm}^{3}, F(000)=$ $1920,2 \theta$ range $1.406-26.451^{\circ}, R$ indices (all data) $R 1=0.0658, w R 2=$ 0.01105 .

\section{2-(6-Bromopyridin-2-yl)-3-(6-bromopyridin-2-ylmethyl)-6-chlo- rochromen-4-one (4c)}

Colorless solid; yield: $418 \mathrm{mg}$ (83\%); $\mathrm{mp} 188-189^{\circ} \mathrm{C}$ (hexanes/EtOAc). ${ }^{1} \mathrm{H} \mathrm{NMR}\left(400 \mathrm{MHz}, \mathrm{CDCl}_{3}\right): \delta=8.15(\mathrm{~d}, J=2.8 \mathrm{~Hz}, 1 \mathrm{H}), 8.06(\mathrm{dd}, J=8.0$, $0.8 \mathrm{~Hz}, 1 \mathrm{H}), 7.72(\mathrm{t}, J=8.0 \mathrm{~Hz}, 1 \mathrm{H}), 7.62(\mathrm{dd}, J=8.8,2.8 \mathrm{~Hz}, 1 \mathrm{H}), 7.56$ $(\mathrm{dd}, J=8.0,0.8 \mathrm{~Hz}, 1 \mathrm{H}), 7.47(\mathrm{~d}, J=8.8 \mathrm{~Hz}, 1 \mathrm{H}), 7.39(\mathrm{t}, J=7.6 \mathrm{~Hz}, 1 \mathrm{H})$, $7.23(\mathrm{~d}, J=7.6 \mathrm{~Hz}, 1 \mathrm{H}), 7.22(\mathrm{~d}, J=7.6 \mathrm{~Hz}, 1 \mathrm{H}), 4.31(\mathrm{~s}, 2 \mathrm{H})$.

${ }^{13} \mathrm{C}\left\{{ }^{1} \mathrm{H}\right\}$ NMR $\left(100 \mathrm{MHz}, \mathrm{CDCl}_{3}\right): \delta=177.1,160.8,158.6,154.1,151.8$, $141.4,141.1,139.0,138.5,134.2,131.1,129.5,125.4,125.3,123.7$, 123.3, 122.0, 120.6, 119.9, 32.8.

HRMS (ESI-TOF): $m / z[\mathrm{M}+\mathrm{H}]^{+}$calcd for $\mathrm{C}_{20} \mathrm{H}_{12} \mathrm{Br}_{2} \mathrm{ClN}_{2} \mathrm{O}_{2}: 504.8954$; found: 504.8950 .

Single-crystal X-ray diagram: crystal of compound $\mathbf{4 c}$ was grown by slow diffusion of EtOAc into a solution of compound $\mathbf{4 c}$ in $\mathrm{CH}_{2} \mathrm{Cl}_{2}$ to yield colorless prisms. The compound crystallizes in the triclinic crystal system, space group $P \overline{1}, a=7.5481(3) \AA, b=10.3357(4) \AA, c=$
13.1031(5) $\AA, V=896.64(6) \AA^{3}, Z=2, d_{\text {calcd }}=1.876 \mathrm{~g} / \mathrm{cm}^{3}, F(000)=496$, $2 \theta$ range $1.682-26.413^{\circ}, R$ indices (all data) $R 1=0.0208, w R 2=$ 0.0475 .

\section{6-Bromo-2-(6-bromopyridin-2-yl)-3-(6-bromopyridin-2-yl- methyl)chromen-4-one (4d)}

Colorless solid; yield: $438 \mathrm{mg}$ (80\%); $\mathrm{mp} 175-176{ }^{\circ} \mathrm{C}$ (hexanes/EtOAc). ${ }^{1} \mathrm{H} \mathrm{NMR}\left(400 \mathrm{MHz}, \mathrm{CDCl}_{3}\right): \delta=8.33(\mathrm{~d}, J=2.4 \mathrm{~Hz}, 1 \mathrm{H}), 8.08(\mathrm{dd}, J=7.6$, $0.8 \mathrm{~Hz}, 1 \mathrm{H}), 7.77$ (dd, $J=8.8,2.4 \mathrm{~Hz}, 1 \mathrm{H}), 7.73(\mathrm{t}, J=8.0 \mathrm{~Hz}, 1 \mathrm{H}), 7.58$ $(\mathrm{dd}, J=8.0,0.4 \mathrm{~Hz}, 1 \mathrm{H}), 7.42(\mathrm{~d}, J=8.8 \mathrm{~Hz}, 1 \mathrm{H}), 7.40(\mathrm{t}, J=8.0 \mathrm{~Hz}, 1 \mathrm{H})$, $7.24(\mathrm{~d}, J=7.6 \mathrm{~Hz}, 1 \mathrm{H}), 7.23(\mathrm{~d}, J=7.6 \mathrm{~Hz}, 1 \mathrm{H}), 4.30(\mathrm{~s}, 2 \mathrm{H})$.

${ }^{13} \mathrm{C}\left\{{ }^{1} \mathrm{H}\right\}$ NMR $\left(100 \mathrm{MHz}, \mathrm{CDCl}_{3}\right): \delta=176.9,160.8,158.6,154.6,151.8$, $141.5,141.1,139.0,138.5,136.9,129.5,128.6,125.4,124.1,123.4$, 122.0, 120.7, 120.1, 118.6, 32.8 .

HRMS (ESI-TOF): $m / z[\mathrm{M}+\mathrm{H}]^{+}$calcd for $\mathrm{C}_{20} \mathrm{H}_{12} \mathrm{Br}_{3} \mathrm{~N}_{2} \mathrm{O}_{2}: 548.8449$; found: 548.8446 .

Single-crystal X-ray diagram: crystal of compound 4d was grown by slow diffusion of EtOAc into a solution of compound $\mathbf{4 d}$ in $\mathrm{CH}_{2} \mathrm{Cl}_{2}$ to yield colorless prisms. The compound crystallizes in the triclinic crystal system, space group $P \overline{1}, a=7.5768(5) \AA, b=10.4119(7) \AA, c=$ 13.2517(9) $\AA, V=915.93(11) \AA^{3}, Z=2, d_{\text {calcd }}=1.998 \mathrm{~g} / \mathrm{cm}^{3}, F(000)=$ $532,2 \theta$ range $1.666-26.479^{\circ}, \mathrm{R}$ indices (all data) $R 1=0.0292, \mathrm{w} R 2=$ 0.0593 .

\section{2-(6-Bromopyridin-2-yl)-3-(6-bromopyridin-2-ylmethyl)-} benzo[h]chromen-4-one (4e)

Colorless solid; yield: $395 \mathrm{mg}$ (76\%); $\mathrm{mp} 223-224^{\circ} \mathrm{C}$ (hexanes/EtOAc). ${ }^{1} \mathrm{H}$ NMR $\left(400 \mathrm{MHz}, \mathrm{CDCl}_{3}\right): \delta=8.50(\mathrm{~d}, J=8.0 \mathrm{~Hz}, 1 \mathrm{H}), 8.19(\mathrm{~d}, J=8.0$ $\mathrm{Hz}, 1 \mathrm{H}), 8.15(\mathrm{~d}, J=8.0 \mathrm{~Hz}, 1 \mathrm{H}), 7.93(\mathrm{~d}, J=7.6 \mathrm{~Hz}, 1 \mathrm{H}), 7.81-7.65(\mathrm{~m}$, $4 \mathrm{H}), 7.60$ (dd, $J=8.0,0.4 \mathrm{~Hz}, 1 \mathrm{H}), 7.40(\mathrm{t}, J=7.6 \mathrm{~Hz}, 1 \mathrm{H}), 7.27$ (d, $J=$ $7.6 \mathrm{~Hz}, 1 \mathrm{H}), 7.23(\mathrm{~d}, J=8.0 \mathrm{~Hz}, 1 \mathrm{H}), 4.44(\mathrm{~s}, 2 \mathrm{H})$.

${ }^{13} \mathrm{C}\left\{{ }^{1} \mathrm{H}\right\}$ NMR $\left(100 \mathrm{MHz}, \mathrm{CDCl}_{3}\right): \delta=177.9,161.2,157.7,152.2,141.5$, $141.1,139.1,138.5,138.7,136.0,129.4,129.3,128.2,127.2,125.5$, $125.2,123.9,123.2,122.4,121.90,121.88,121.0,119.2,32.9$.

HRMS (ESI-TOF): $m / z[\mathrm{M}+\mathrm{H}]^{+}$calcd for $\mathrm{C}_{24} \mathrm{H}_{15} \mathrm{Br}_{2} \mathrm{~N}_{2} \mathrm{O}_{2}: 520.9500$; found: 520.9496 .

\section{2-(6-Bromopyridin-2-yl)-3-(6-bromopyridin-2-ylmethyl)-7- methoxychromen-4-one (4f)}

Colorless solid; yield: $360 \mathrm{mg}$ (72\%); mp 198-199 ${ }^{\circ} \mathrm{C}$ (hexanes/EtOAc). ${ }^{1} \mathrm{H} \mathrm{NMR}\left(400 \mathrm{MHz}, \mathrm{CDCl}_{3}\right): \delta=8.12(\mathrm{~d}, J=8.8 \mathrm{~Hz}, 1 \mathrm{H}), 8.07(\mathrm{dd}, J=7.6$, $0.8 \mathrm{~Hz}, 1 \mathrm{H}), 7.72(\mathrm{t}, J=8.0 \mathrm{~Hz}, 1 \mathrm{H}), 7.56(\mathrm{dd}, J=8.0,0.8 \mathrm{~Hz}, 1 \mathrm{H}), 7.39$ $(\mathrm{t}, J=7.6 \mathrm{~Hz}, 1 \mathrm{H}), 7.25(\mathrm{~d}, J=8.8 \mathrm{~Hz}, 1 \mathrm{H}), 7.23(\mathrm{~d}, J=7.6 \mathrm{~Hz}, 1 \mathrm{H}), 6.98$ (dd, $J=8.8,2.4 \mathrm{~Hz}, 1 \mathrm{H}), 6.91(\mathrm{~d}, J=2.0 \mathrm{~Hz}, 1 \mathrm{H}), 4.28(\mathrm{~s}, 2 \mathrm{H}), 3.91(\mathrm{~s}, 3$ $\mathrm{H})$.

${ }^{13} \mathrm{C}\left\{{ }^{1} \mathrm{H}\right\}$ NMR $\left(100 \mathrm{MHz}, \mathrm{CDCl}_{3}\right): \delta=177.4,164.4,161.3,158.1,147.7$, $152.2,141.5,141.0,138.9,138.5,129.3,127.4,125.2,123.3,121.9$, 120.3, 116.9, 114.9, 100.1, 55.9, 32.9.

HRMS (ESI-TOF): $m / z[\mathrm{M}+\mathrm{H}]^{+}$calcd for $\mathrm{C}_{21} \mathrm{H}_{15} \mathrm{Br}_{2} \mathrm{~N}_{2} \mathrm{O}_{3}$ : 500.9450; found: 500.9444 .

\section{2-(6-Bromopyridin-2-yl)-3-(6-bromopyridin-2-ylmethyl)-6- methoxychromen-4-one $(4 \mathrm{~g})$}

Colorless solid; yield: $400 \mathrm{mg}$ (80\%); $\mathrm{mp} 177-178^{\circ} \mathrm{C}$ (hexanes/EtOAc). ${ }^{1} \mathrm{H}$ NMR $\left(400 \mathrm{MHz}, \mathrm{CDCl}_{3}\right): \delta=8.01(\mathrm{~d}, J=7.6 \mathrm{~Hz}, 1 \mathrm{H}), 7.68(\mathrm{t}, J=7.6$ $\mathrm{Hz}, 1 \mathrm{H}), 7.52-7.50(\mathrm{~m}, 2 \mathrm{H}), 7.41(\mathrm{~d}, J=9.2 \mathrm{~Hz}, 1 \mathrm{H}), 7.36(\mathrm{t}, J=7.6 \mathrm{~Hz}$, $1 \mathrm{H}), 7.25(\mathrm{dd}, J=8.8,2.8 \mathrm{~Hz}, 1 \mathrm{H}), 7.20(\mathrm{~d}, J=7.6 \mathrm{~Hz}, 2 \mathrm{H}), 4.32(\mathrm{~s}, 2 \mathrm{H})$, $3.84(\mathrm{~s}, 3 \mathrm{H})$. 
${ }^{13} \mathrm{C}\left\{{ }^{1} \mathrm{H}\right\}$ NMR $\left(100 \mathrm{MHz}, \mathrm{CDCl}_{3}\right): \delta=177.9,161.3,158.0,156.9,152.0$, 150.6, 141.2, 140.9, 138.9, 138.4, 129.2, 125.1, 124.1, 123.2, 123.1, 121.7, 119.50, 119.45, 104.7, 55.8, 32.9.

HRMS (ESI-TOF): $m / z[\mathrm{M}+\mathrm{H}]^{+}$calcd for $\mathrm{C}_{21} \mathrm{H}_{15} \mathrm{Br}_{2} \mathrm{~N}_{2} \mathrm{O}_{3}$ : 500.9450; found: 500.9444 .

Single-crystal X-ray diagram: crystal of compound $\mathbf{4 g}$ was grown by slow diffusion of EtOAc into a solution of compound $4 \mathrm{~g}$ in $\mathrm{CH}_{2} \mathrm{Cl}_{2}$ to yield colorless prisms. The compound crystallizes in the monoclinic crystal system, space group $P 21 / c, a=4.4683(4) \AA$, $b=22.3901(19) \AA$, $c=18.7910(16) \AA, V=1879.6(3) \AA^{3}, Z=4, d_{\text {calcd }}=1.774 \mathrm{~g} / \mathrm{cm}^{3}, F(000)=$ 992, $2 \theta$ range $1.415-26.411^{\circ}, R$ indices (all data) $R 1=0.0443, w R 2=$ 0.0972 .

2-(6-Bromopyridin-2-yl)-3-(6-bromopyridin-2-ylmethyl)-5methoxychromen-4-one (4h)

Colorless solid; yield: $350 \mathrm{mg}$ (70\%); mp $187-188^{\circ} \mathrm{C}$ (hexanes/EtOAc). ${ }^{1} \mathrm{H}$ NMR $\left(400 \mathrm{MHz}, \mathrm{CDCl}_{3}\right): \delta=8.08(\mathrm{~d}, J=7.6 \mathrm{~Hz}, 1 \mathrm{H}), 7.71(\mathrm{t}, J=7.6$ $\mathrm{Hz}, 1 \mathrm{H}), 7.58(\mathrm{~d}, J=8.4 \mathrm{~Hz}, 1 \mathrm{H}), 7.56(\mathrm{~d}, J=8.4 \mathrm{~Hz}, 1 \mathrm{H}), 7.37(\mathrm{t}, J=7.6$ $\mathrm{Hz}, 1 \mathrm{H}), 7.26(\mathrm{~d}, J=7.6 \mathrm{~Hz}, 1 \mathrm{H}), 7.21(\mathrm{~d}, J=8.0 \mathrm{~Hz}, 1 \mathrm{H}), 7.07(\mathrm{dd}, J=$ 8.4, 0.8 Hz, $1 \mathrm{H}), 6.81$ (d, J = 8.0 Hz, $1 \mathrm{H}), 4.23(\mathrm{~s}, 2 \mathrm{H}), 3.97(\mathrm{~s}, 3 \mathrm{H})$.

${ }^{13} \mathrm{C}\left\{{ }^{1} \mathrm{H}\right\}$ NMR $\left(100 \mathrm{MHz}, \mathrm{CDCl}_{3}\right): \delta=177.7,161.4,159.8,157.9,156.8$, 152.0, 141.4, 140.9, 138.9, 138.5, 134.0, 129.2, 125.1, 123.3, 122.1, 121.5, 113.6, 110.1, 106.2, 56.4, 32.9.

HRMS (ESI-TOF): $m / z[\mathrm{M}+\mathrm{H}]^{+}$calcd for $\mathrm{C}_{21} \mathrm{H}_{15} \mathrm{Br}_{2} \mathrm{~N}_{2} \mathrm{O}_{3}$ : 500.9450; found: 500.9445 .

\section{2-(6-Bromopyridin-2-yl)-3-(6-bromopyridin-2-ylmethyl)-7- butoxychromen-4-one ( $4 \mathrm{i})$}

Colorless solid; yield: $450 \mathrm{mg}$ (83\%); mp $140-141{ }^{\circ} \mathrm{C}$ (hexanes/EtOAc). ${ }^{1} \mathrm{H} \mathrm{NMR}\left(400 \mathrm{MHz}, \mathrm{CDCl}_{3}\right): \delta=8.10(\mathrm{~d}, J=9.2 \mathrm{~Hz}, 1 \mathrm{H}), 8.06(\mathrm{dd}, J=8.0$, $0.8 \mathrm{~Hz}, 1 \mathrm{H}), 7.71(\mathrm{t}, J=8.0 \mathrm{~Hz}, 1 \mathrm{H}), 7.56(\mathrm{dd}, J=8.0,0.8 \mathrm{~Hz}, 1 \mathrm{H}), 7.39$ $(\mathrm{t}, J=7.6 \mathrm{~Hz}, 1 \mathrm{H}), 7.24(\mathrm{t}, J=7.6 \mathrm{~Hz}, 1 \mathrm{H}), 7.23(\mathrm{~d}, J=7.6 \mathrm{~Hz}, 1 \mathrm{H}), 6.97$ (dd, $J=8.8,2.4 \mathrm{~Hz}, 1 \mathrm{H}), 6.89(\mathrm{~d}, J=2.4 \mathrm{~Hz}, 1 \mathrm{H}), 4.28(\mathrm{~s}, 2 \mathrm{H}), 4.05(\mathrm{t}$, $J=6.4 \mathrm{~Hz}, 2 \mathrm{H}), 1.86-1.79(\mathrm{~m}, 2 \mathrm{H}), 1.56-1.49$ (m, $2 \mathrm{H}), 0.99$ (t, $J=7.6$ $\mathrm{Hz}, 3 \mathrm{H})$.

${ }^{13} \mathrm{C}\left\{{ }^{1} \mathrm{H}\right\}$ NMR $\left(100 \mathrm{MHz}, \mathrm{CDCl}_{3}\right): \delta=177.4,163.9,161.4,158.1,157.7$, $152.2,141.4,141.0,138.9,138.5,129.2,127.3,125.2,123.3,121.9$, 120.3, 116.7, 115.3, 100.5, 68.5, 32.9, 31.0, 19.2, 13.8 .

HRMS (ESI-TOF): $m / z[\mathrm{M}+\mathrm{H}]^{+}$calcd for $\mathrm{C}_{24} \mathrm{H}_{21} \mathrm{Br}_{2} \mathrm{~N}_{2} \mathrm{O}_{3}$ : 542.9919; found: 542.9916 .

\section{2-(6-Bromopyridin-2-yl)-3-(6-bromopyridin-2-ylmethyl)-6- butoxychromen-4-one $(4 \mathbf{j})$}

Colorless solid; yield: $434 \mathrm{mg}$ (80\%); $\mathrm{mp} 124-125^{\circ} \mathrm{C}$ (hexanes/EtOAc). ${ }^{1} \mathrm{H} \mathrm{NMR}\left(400 \mathrm{MHz}, \mathrm{CDCl}_{3}\right): \delta=8.05(\mathrm{~d}, J=8.4 \mathrm{~Hz}, 1 \mathrm{H}), 7.72(\mathrm{t}, J=8.0$ $\mathrm{Hz}, 1 \mathrm{H}), 7.56(\mathrm{~d}, J=8.8 \mathrm{~Hz}, 1 \mathrm{H}), 7.55(\mathrm{~s}, 1 \mathrm{H}), 7.45(\mathrm{~d}, J=9.2 \mathrm{~Hz}, 1 \mathrm{H})$, $7.38(\mathrm{t}, J=7.6 \mathrm{~Hz}, 1 \mathrm{H}), 7.29(\mathrm{dd}, J=9.2,3.2 \mathrm{~Hz}, 1 \mathrm{H}), 7.24(\mathrm{~d}, J=8.0 \mathrm{~Hz}$, $2 \mathrm{H}), 4.32(\mathrm{~s}, 2 \mathrm{H}), 4.05(\mathrm{t}, J=6.4 \mathrm{~Hz}, 2 \mathrm{H}), 1.83-1.76(\mathrm{~m}, 2 \mathrm{H}), 1.55-$ $1.45(\mathrm{~m}, 2 \mathrm{H}), 0.98(\mathrm{t}, J=7.6 \mathrm{~Hz}, 3 \mathrm{H})$.

${ }^{13} \mathrm{C}\left\{{ }^{1} \mathrm{H}\right\} \operatorname{NMR}\left(100 \mathrm{MHz}, \mathrm{CDCl}_{3}\right): \delta=178.1,161.4,158.2,156.6,152.3$, $150.6,141.4,141.0,139.9,138.5,129.3,125.2,124.6,123.4,123.3$, $121.8,119.6,119.5,106.5,68.4,33.0,31.1,19.2,13.8$.

HRMS (ESI-TOF): $m / z[\mathrm{M}+\mathrm{H}]^{+}$calcd for $\mathrm{C}_{24} \mathrm{H}_{21} \mathrm{Br}_{2} \mathrm{~N}_{2} \mathrm{O}_{3}$ : 542.9919; found: 542.9916 .

2-(6-Bromopyridin-2-yl)-3-(6-bromopyridin-2-ylmethyl)-5butoxychromen-4-one (4k)

Colorless solid; yield: $347 \mathrm{mg}$ (64\%); mp $170-171^{\circ} \mathrm{C}$ (hexanes/EtOAc).
${ }^{1} \mathrm{H} \mathrm{NMR}\left(400 \mathrm{MHz}, \mathrm{CDCl}_{3}\right): \delta=8.01(\mathrm{~d}, J=8.4 \mathrm{~Hz}, 1 \mathrm{H}), 7.69(\mathrm{t}, J=7.6$ $\mathrm{Hz}, 1 \mathrm{H}), 7.55$ (d, J = 8.4 Hz, $1 \mathrm{H}), 7.53(\mathrm{~d}, J=8.4 \mathrm{~Hz}, 1 \mathrm{H}), 7.35$ (d, $J=7.6$ $\mathrm{Hz}, 1 \mathrm{H}), 7.21(\mathrm{~d}, J=7.6 \mathrm{~Hz}, 1 \mathrm{H}), 7.20(\mathrm{~d}, J=7.6 \mathrm{~Hz}, 1 \mathrm{H}), 7.04(\mathrm{~d}, J=8.4$ $\mathrm{Hz}, 1 \mathrm{H}), 6.80(\mathrm{~d}, J=8.4 \mathrm{~Hz}, 1 \mathrm{H}), 4.29(\mathrm{~s}, 2 \mathrm{H}), 4.10(\mathrm{t}, J=6.4 \mathrm{~Hz}, 2 \mathrm{H})$, $1.95-1.88$ (m, 2 H), 1.60-1.50 (m, $2 \mathrm{H}), 0.98$ (t, J = 7.6 Hz, $3 \mathrm{H}$ ).

${ }^{13} \mathrm{C}\left\{{ }^{1} \mathrm{H}\right\}$ NMR $\left(100 \mathrm{MHz}, \mathrm{CDCl}_{3}\right): \delta=177.4,161.6,159.4,157.8,156.4$, $152.1,141.3,140.9,138.8,138.4,133.9,129.1,125.0,123.0,121.8$, $121.3,113.8,109.7,107.3,69.3,32.7,31.0,19.2,13.8$.

HRMS (ESI-TOF): $m / z[\mathrm{M}+\mathrm{H}]^{+}$calcd for $\mathrm{C}_{24} \mathrm{H}_{21} \mathrm{Br}_{2} \mathrm{~N}_{2} \mathrm{O}_{3}$ : 542.9919; found: 542.9915 .

\section{7-Benzyloxy-2-(6-bromopyridin-2-yl)-3-(6-bromopyridin-2-yl- methyl)chromen-4-one (41)}

Colorless solid; yield: $461 \mathrm{mg}(80 \%)$; $\mathrm{mp} 216-217^{\circ} \mathrm{C}$ (hexanes/EtOAc). ${ }^{1} \mathrm{H} \mathrm{NMR}\left(400 \mathrm{MHz}, \mathrm{CDCl}_{3}\right): \delta=8.13(\mathrm{~d}, J=9.2 \mathrm{~Hz}, 1 \mathrm{H}), 8.06(\mathrm{~d}, J=7.6$ $\mathrm{Hz}, 1 \mathrm{H}), 7.71(\mathrm{t}, J=8.0 \mathrm{~Hz}, 1 \mathrm{H}), 7.56(\mathrm{dd}, J=8.0,0.8 \mathrm{~Hz}, 1 \mathrm{H}), 7.46-$ $7.34(\mathrm{~m}, 6 \mathrm{H}), 7.24$ (d, $J=7.6 \mathrm{~Hz}, 2 \mathrm{H}), 7.06$ (dd, $J=8.8,2.4 \mathrm{~Hz}, 1 \mathrm{H}$ ), $6.98(\mathrm{~d}, J=2.4 \mathrm{~Hz}, 1 \mathrm{H}), 5.17(\mathrm{~s}, 2 \mathrm{H}), 4.28(\mathrm{~s}, 2 \mathrm{H})$.

${ }^{13} \mathrm{C}\left\{{ }^{1} \mathrm{H}\right\}$ NMR $\left(100 \mathrm{MHz}, \mathrm{CDCl}_{3}\right): \delta=177.4,163.4,161.3,158.2,157.6$, $152.1,141.4,141.0,138.9,138.5,135.6,129.3,128.8(2 \times), 128.4$, 127.52, $127.47(2 \times), 125.2,123.3,121.9,120.4,117.0,115.4,101.1$, 70.5, 32.9.

HRMS (ESI-TOF): $m / z[M+H]^{+}$calcd for $\mathrm{C}_{27} \mathrm{H}_{19} \mathrm{Br}_{2} \mathrm{~N}_{2} \mathrm{O}_{3}: 576.9763$; found: 576.9757 .

\section{6-Benzyloxy-2-(6-bromopyridin-2-yl)-3-(6-bromopyridin-2-yl- methyl)chromen-4-one (4m)}

Colorless solid; yield: $420 \mathrm{mg}$ (73\%); $\mathrm{mp} 184-185^{\circ} \mathrm{C}$ (hexanes/EtOAc). ${ }^{1} \mathrm{H} \mathrm{NMR}\left(400 \mathrm{MHz}, \mathrm{CDCl}_{3}\right): \delta=8.05(\mathrm{dd}, J=7.6,0.4 \mathrm{~Hz}, 1 \mathrm{H}), 7.72(\mathrm{t}, J=$ $7.6 \mathrm{~Hz}, 1 \mathrm{H}), 7.68(\mathrm{~d}, J=2.8 \mathrm{~Hz}, 1 \mathrm{H}), 7.56(\mathrm{dd}, J=8.0,0.8 \mathrm{~Hz}, 1 \mathrm{H})$, 7.48-7.34 (m, $8 \mathrm{H}$ ), 7.24 (d, J = 7.6 Hz, $2 \mathrm{H}$ ), 5.14 (s, $2 \mathrm{H}), 4.33$ (s, $2 \mathrm{H}$ ).

${ }^{13} \mathrm{C}\left\{{ }^{1} \mathrm{H}\right\}$ NMR $\left(100 \mathrm{MHz}, \mathrm{CDCl}_{3}\right): \delta=178.0,161.3,158.3,156.1,152.2$, $150.8,141.4,141.0,138.9,138.5,136.2,129.3,128.6(2 \times), 128.2$, $127.7(2 \times), 125.2,124.7,123.4,123.3,121.8,119.7,119.6,106.1,70.6$, 33.0.

HRMS (ESI-TOF): $m / z[\mathrm{M}+\mathrm{H}]^{+}$calcd for $\mathrm{C}_{27} \mathrm{H}_{19} \mathrm{Br}_{2} \mathrm{~N}_{2} \mathrm{O}_{3}$ : 576.9763; found: 576.9757 .

\section{5-Benzyloxy-2-(6-bromopyridin-2-yl)-3-(6-bromopyridin-2-yl- methyl)chromen-4-one (4n)}

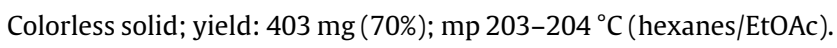
${ }^{1} \mathrm{H} \mathrm{NMR}\left(400 \mathrm{MHz}, \mathrm{CDCl}_{3}\right): \delta=8.03(\mathrm{dd}, J=7.6,0.4 \mathrm{~Hz}, 1 \mathrm{H}), 7.70(\mathrm{t}, J=$ $8.0 \mathrm{~Hz}, 1 \mathrm{H}), 7.57-7.53(\mathrm{~m}, 3 \mathrm{H}), 7.51(\mathrm{t}, J=8.0 \mathrm{~Hz}, 1 \mathrm{H}), 7.40-7.36(\mathrm{~m}$, $3 \mathrm{H}), 7.29(\mathrm{t}, J=7.2 \mathrm{~Hz}, 1 \mathrm{H}), 7.23(\mathrm{dt}, J=8.0,0.4 \mathrm{~Hz}, 1 \mathrm{H}), 7.07$ (dd, $J=$ 8.4, 0.4 Hz, $2 \mathrm{H}), 6.83(\mathrm{~d}, J=8.0 \mathrm{~Hz}, 1 \mathrm{H}), 5.30(\mathrm{~s}, 2 \mathrm{H}), 4.32(\mathrm{~s}, 2 \mathrm{H})$.

${ }^{13} \mathrm{C}\left\{{ }^{1} \mathrm{H}\right\}$ NMR $\left(100 \mathrm{MHz}, \mathrm{CDCl}_{3}\right): \delta=177.4,161.5,158.6,157.9,156.5$, $152.1,141.4,140.9,138.9,138.5,136.4,133.8,129.2,128.6(2 \times)$, 127.7, $126.7(2 \times), 125.1,123.1,121.9,121.5,114.2,110.4,108.4,70.9$, 32.8 .

HRMS (ESI-TOF): $m / z[M+H]^{+}$calcd for $\mathrm{C}_{27} \mathrm{H}_{19} \mathrm{Br}_{2} \mathrm{~N}_{2} \mathrm{O}_{3}$ : 576.9763; found: 576.9758 .

2-(6-Bromopyridin-2-yl)-3-(6-bromopyridin-2-ylmethyl)-6,8-dichlorochromen-4-one (40)

Colorless solid; yield: $420 \mathrm{mg}$ (78\%); $\mathrm{mp} 216-217^{\circ} \mathrm{C}$ (hexanes/EtOAc). 
${ }^{1} \mathrm{H} \mathrm{NMR}\left(400 \mathrm{MHz}, \mathrm{CDCl}_{3}\right): \delta=8.13(\mathrm{dd}, J=7.6,0.8 \mathrm{~Hz}, 1 \mathrm{H}), 8.11(\mathrm{~d}, J=$ $2.4 \mathrm{~Hz}, 1 \mathrm{H}), 7.76(\mathrm{t}, J=8.0 \mathrm{~Hz}, 1 \mathrm{H}), 7.75(\mathrm{~d}, J=8.8 \mathrm{~Hz}, 1 \mathrm{H}), 7.57$ (dd, $J=8.0,0.8 \mathrm{~Hz}, 1 \mathrm{H}), 7.38(\mathrm{t}, J=8.0 \mathrm{~Hz}, 1 \mathrm{H}), 7.22(\mathrm{~d}, J=8.0 \mathrm{~Hz}, 1 \mathrm{H})$, 7.20 (dd, J = 7.6, $0.8 \mathrm{~Hz}, 1 \mathrm{H}), 4.51(\mathrm{~s}, 2 \mathrm{H})$.

${ }^{13} \mathrm{C}\left\{{ }^{1} \mathrm{H}\right\}$ NMR $\left(100 \mathrm{MHz}, \mathrm{CDCl}_{3}\right): \delta=176.7,160.7,157.8,151.7,150.1$, 141.22, 141.16, 139.2, 138.4, 134.0, 130.9, 129.7, 125.3, 124.4, 124.3, 124.2, 123.1, 121.7, 121.1, 32.5.

HRMS (ESI-TOF): $m / z[\mathrm{M}+\mathrm{H}]^{+}$calcd for $\mathrm{C}_{20} \mathrm{H}_{11} \mathrm{Br}_{2} \mathrm{Cl}_{2} \mathrm{~N}_{2} \mathrm{O}_{2}$ : 538.8564; found: 538.8560 .

\section{2-(6-Bromopyridin-2-yl)-3-(6-bromopyridin-2-ylmethyl)-6,8-di- fluorochromen-4-one (4p)}

Colorless solid; yield: $405 \mathrm{mg}$ (80\%); $\mathrm{mp} 155-156{ }^{\circ} \mathrm{C}$ (hexanes/EtOAc). ${ }^{1} \mathrm{H} \mathrm{NMR}\left(400 \mathrm{MHz}, \mathrm{CDCl}_{3}\right.$ ): $\delta=8.09$ (dd, $J=7.6,0.8 \mathrm{~Hz}, 1 \mathrm{H}$ ), $7.74(\mathrm{t}, J=$ $8.0 \mathrm{~Hz}, 1 \mathrm{H}), 7.65(\mathrm{dt}, J=8.0,2.8 \mathrm{~Hz}, 1 \mathrm{H}), 7.57(\mathrm{dd}, J=8.0,0.8 \mathrm{~Hz}, 1 \mathrm{H})$, $7.39(\mathrm{t}, J=7.6 \mathrm{~Hz}, 1 \mathrm{H}), 7.27(\mathrm{dt}, J=8.0,2.8 \mathrm{~Hz}, 1 \mathrm{H}), 7.20(\mathrm{~d}, J=7.6 \mathrm{~Hz}$, $2 \mathrm{H}), 4.41$ ( $\mathrm{s}, 2 \mathrm{H})$.

${ }^{13} \mathrm{C}\left\{{ }^{1} \mathrm{H}\right\}$ NMR $\left(100 \mathrm{MHz}, \mathrm{CDCl}_{3}\right): \delta=176.5(\mathrm{t}, J=3.1 \mathrm{~Hz}), 160.6,158.3$ (dd, $J=9.8,247.9 \mathrm{~Hz}), 158.0,151.6,151.4(\mathrm{dd}, J=11.4,256.2 \mathrm{~Hz}$ ), $141.4(\mathrm{~d}, J=2.3 \mathrm{~Hz}), 141.3,141.1,139.1,138.5,129.7,125.3,124.8$ (d, $J=8.3 \mathrm{~Hz}$ ), 123.2, 121.8, 120.6, 109.3 (dd, $J=20.5,28.8 \mathrm{~Hz}$ ), 106.2 (dd, $J=4.6,23.5 \mathrm{~Hz}), 32.5$.

HRMS (ESI-TOF): $m / z[\mathrm{M}+\mathrm{H}]^{+}$calcd for $\mathrm{C}_{20} \mathrm{H}_{11} \mathrm{Br}_{2} \mathrm{~F}_{2} \mathrm{~N}_{2} \mathrm{O}_{2}$ : 506.9155; found: 506.9151 .

\section{2-(6-Bromopyridin-2-yl)-3-(6-bromopyridin-2-ylmethyl)-6-meth-} ylchromen-4-one (4q)

Colorless solid; yield: $402 \mathrm{mg}$ (83\%); mp $163-164{ }^{\circ} \mathrm{C}$ (hexanes/EtOAc). ${ }^{1} \mathrm{H} \mathrm{NMR}\left(400 \mathrm{MHz}, \mathrm{CDCl}_{3}\right): \delta=8.02(\mathrm{~d}, J=7.6 \mathrm{~Hz}, 1 \mathrm{H}), 7.97(\mathrm{t}, J=0.4$ $\mathrm{Hz}, 1 \mathrm{H}), 7.70(\mathrm{t}, J=7.6 \mathrm{~Hz}, 1 \mathrm{H}), 7.53(\mathrm{~d}, J=8.0 \mathrm{~Hz}, 1 \mathrm{H}), 7.49(\mathrm{dd}, J=$ 8.4, 2.0 Hz, $1 \mathrm{H}$ ), 7.39 (d, J = 8.4 Hz, $1 \mathrm{H}), 7.35$ (d, J = 7.6 Hz, $1 \mathrm{H}), 7.22$ $(\mathrm{d}, J=8.0 \mathrm{~Hz}, 2 \mathrm{H}), 4.32(\mathrm{~s}, 2 \mathrm{H}), 2.43(\mathrm{~s}, 3 \mathrm{H})$.

${ }^{13} \mathrm{C}\left\{{ }^{1} \mathrm{H}\right\}$ NMR $\left(100 \mathrm{MHz}, \mathrm{CDCl}_{3}\right): \delta=178.2,161.3,158.2,154.1,152.1$, 141.3, 141.0, 138.9, 138.4, 135.23, 135.20, 129.2, 125.2, 125.1, 123.2, 122.4, 121.7, 120.2, 117.8, 32.9, 20.9.

HRMS (ESI-TOF): $m / z[\mathrm{M}+\mathrm{H}]^{+}$calcd for $\mathrm{C}_{21} \mathrm{H}_{15} \mathrm{Br}_{2} \mathrm{~N}_{2} \mathrm{O}_{2}: 484.9500$; found: 484.9493 .

\section{3-(6-Bromopyridin-2-yl)-2-(6-bromopyridin-2-ylmethyl)- benzo[f]chromen-1-one (4r)}

Colorless solid; yield: $348 \mathrm{mg}$ (67\%); mp $177-178^{\circ} \mathrm{C}$ (hexanes/EtOAc). ${ }^{1} \mathrm{H}$ NMR $\left(400 \mathrm{MHz}, \mathrm{CDCl}_{3}\right): \delta=10.03(\mathrm{~d}, J=8.0 \mathrm{~Hz}, 1 \mathrm{H}), 8.14(\mathrm{dd}, J=$ 8.4, $0.8 \mathrm{~Hz}, 1 \mathrm{H}), 8.12(\mathrm{~d}, J=7.6 \mathrm{~Hz}, 1 \mathrm{H}), 7.92(\mathrm{~d}, J=8.0 \mathrm{~Hz}, 1 \mathrm{H}), 7.75$ $(\mathrm{t}, J=8.0 \mathrm{~Hz}, 1 \mathrm{H}), 7.74(\mathrm{t}, J=8.0 \mathrm{~Hz}, 1 \mathrm{H}), 7.63(\mathrm{dt}, J=8.0,1.2 \mathrm{~Hz}, 1 \mathrm{H})$, $7.59(\mathrm{~d}, J=8.8 \mathrm{~Hz}, 1 \mathrm{H}), 7.58(\mathrm{dd}, J=7.6,0.8 \mathrm{~Hz}, 1 \mathrm{H}), 7.41(\mathrm{~d}, J=7.6 \mathrm{~Hz}$, $1 \mathrm{H}), 7.29(\mathrm{~d}, J=8.0 \mathrm{~Hz}, 1 \mathrm{H}), 7.25(\mathrm{~d}, J=7.6 \mathrm{~Hz}, 1 \mathrm{H}), 4.42(\mathrm{~s}, 2 \mathrm{H})$.

${ }^{13} \mathrm{C}\left\{{ }^{1} \mathrm{H}\right\}$ NMR $\left(100 \mathrm{MHz}, \mathrm{CDCl}_{3}\right): \delta=179.5,161.4,157.1,156.3,152.0$, $141.5,141.1,139.0,138.6,135.9,130.6,130.4,129.4,129.3,128.3$, 127.1, 126.7, 125.2, 123.3, 123.0, 121.8, 117.6, 116.2, 33.2.

HRMS (ESI-TOF): $m / z[\mathrm{M}+\mathrm{H}]^{+}$calcd for $\mathrm{C}_{24} \mathrm{H}_{15} \mathrm{Br}_{2} \mathrm{~N}_{2} \mathrm{O}_{2}$ : 520.9500; found: 520.9495 .

\section{2-(6-Bromopyridin-2-yl)-3-(6-bromopyridin-2-ylmethyl)-6-} phenylchromen-4-one (4s)

Colorless solid; yield: $437 \mathrm{mg}(80 \%)$; $\mathrm{mp} 174-175^{\circ} \mathrm{C}$ (hexanes/EtOAc).
${ }^{1} \mathrm{H}$ NMR $\left(400 \mathrm{MHz}, \mathrm{CDCl}_{3}\right): \delta=8.43(\mathrm{~d}, J=2.4 \mathrm{~Hz}, 1 \mathrm{H}), 8.09(\mathrm{dd}, J=7.6$, $0.8 \mathrm{~Hz}, 1 \mathrm{H}), 7.94(\mathrm{dd}, J=8.4,2.4 \mathrm{~Hz}, 1 \mathrm{H}), 7.73(\mathrm{t}, J=7.6 \mathrm{~Hz}, 1 \mathrm{H}), 7.67-$ $7.64(\mathrm{~m}, 2 \mathrm{H}), 7.583(\mathrm{t}, J=8.0 \mathrm{~Hz}, 1 \mathrm{H}), 7.580(\mathrm{t}, J=8.0 \mathrm{~Hz}, 1 \mathrm{H}), 7.48-$ $7.44(\mathrm{~m}, 2 \mathrm{H}), 7.42-7.36(\mathrm{~m}, 2 \mathrm{H}), 7.26(\mathrm{~d}, J=8.4 \mathrm{~Hz}, 1 \mathrm{H}), 7.24(\mathrm{dd}, J=$ 8.0, $0.4 \mathrm{~Hz}, 1 \mathrm{H}), 4.36(\mathrm{~s}, 2 \mathrm{H})$.

${ }^{13} \mathrm{C}\left\{{ }^{1} \mathrm{H}\right\}$ NMR $\left(100 \mathrm{MHz}, \mathrm{CDCl}_{3}\right): \delta=178.2,161.2,158.4,155.3,152.1$, $141.4,141.1,139.2,139.0,138.5,138.4,132.9,129.4,129.0(2 \times)$, 127.8, 127.1 (2×), 125.2, 123.8, 123.3, 122.9, 121.9, 120.5, 118.6, 32.9. HRMS (ESI-TOF): $m / z[\mathrm{M}+\mathrm{H}]^{+}$calcd for $\mathrm{C}_{26} \mathrm{H}_{17} \mathrm{Br}_{2} \mathrm{~N}_{2} \mathrm{O}_{2}: 546.9657$; found: 546.9652 .

\section{2-(6-Bromopyridin-2-yl)-3-(6-bromopyridin-2-ylmethyl)-6-(4- fluorophenyl)chromen-4-one (4t)}

Colorless solid; yield: $440 \mathrm{mg}$ (78\%); $\mathrm{mp} 191-192^{\circ} \mathrm{C}$ (hexanes/EtOAc). ${ }^{1} \mathrm{H} \mathrm{NMR}\left(400 \mathrm{MHz}, \mathrm{CDCl}_{3}\right): \delta=8.37(\mathrm{~d}, J=2.4 \mathrm{~Hz}, 1 \mathrm{H}), 8.09(\mathrm{dd}, J=7.6$, $0.8 \mathrm{~Hz}, 1 \mathrm{H}), 7.88(\mathrm{dd}, J=8.4,2.4 \mathrm{~Hz}, 1 \mathrm{H}), 7.73(\mathrm{t}, J=7.6 \mathrm{~Hz}, 1 \mathrm{H}), 7.62-$ $7.56(\mathrm{~m}, 4 \mathrm{H}), 7.40(\mathrm{t}, J=7.6 \mathrm{~Hz}, 1 \mathrm{H}), 7.26(\mathrm{~d}, J=7.6 \mathrm{~Hz}, 1 \mathrm{H}), 7.24(\mathrm{~d}$, $J=7.6 \mathrm{~Hz}, 1 \mathrm{H}), 7.18-7.13(\mathrm{~m}, 2 \mathrm{H}), 4.35$ (s, $2 \mathrm{H})$.

${ }^{13} \mathrm{C}\left\{{ }^{1} \mathrm{H}\right\}$ NMR $\left(100 \mathrm{MHz}, \mathrm{CDCl}_{3}\right): \delta=178.2,162.7(\mathrm{~d}, J=246.3 \mathrm{~Hz})$, $161.1,158.5,155.2,152.0,141.4,141.1,139.0,138.5,137.4,135.4$ (d, $J=3.1 \mathrm{~Hz}), 132.7,129.4,128.8(\mathrm{~d}, J=8.4 \mathrm{~Hz}, 2 \times), 125.3,123.7,123.3$, 122.9, 121.9, 120.5, 118.7, $115.9(\mathrm{~d}, J=21.2 \mathrm{~Hz}, 2 \times), 32.9$.

HRMS (ESI-TOF): $m / z[\mathrm{M}+\mathrm{H}]^{+}$calcd for $\mathrm{C}_{26} \mathrm{H}_{16} \mathrm{Br}_{2} \mathrm{FN}_{2} \mathrm{O}_{2}: 564.9563$; found: 564.9557 .

Single-crystal X-ray diagram: crystal of compound $\mathbf{4 t}$ was grown by slow diffusion of EtOAc into a solution of compound $4 \mathbf{t}$ in $\mathrm{CH}_{2} \mathrm{Cl}_{2}$ to yield colorless prisms. The compound crystallizes in the triclinic crystal system, space group $P \overline{1}, a=7.4478(6) \AA, b=10.0187(8) \AA, c=$ $15.9674(13) \AA \AA, V=1079.68(15) \AA^{3}, Z=2, d_{\text {calcd }}=1.742 \mathrm{~g} / \mathrm{cm}^{3}, F(000)=$ $560,2 \theta$ range $1.315-26.402^{\circ}, R$ indices (all data) $R 1=0.0221, w R 2=$ 0.0517 .

\section{2-(6-Bromopyridin-2-yl)-3-(6-bromopyridin-2-ylmethyl)-6-(4-} methoxyphenyl)chromen-4-one (4u)

Colorless solid; yield: $449 \mathrm{mg}$ (78\%); $\mathrm{mp} 162-163^{\circ} \mathrm{C}$ (hexanes/EtOAc). ${ }^{1} \mathrm{H} \mathrm{NMR}\left(400 \mathrm{MHz}, \mathrm{CDCl}_{3}\right): \delta=8.36(\mathrm{~d}, J=2.4 \mathrm{~Hz}, 1 \mathrm{H}), 8.07(\mathrm{~d}, J=7.2$ $\mathrm{Hz}, 1 \mathrm{H}), 7.89$ (dd, $J=8.4,2.4 \mathrm{~Hz}, 1 \mathrm{H}), 7.72(\mathrm{t}, J=7.6 \mathrm{~Hz}, 1 \mathrm{H}), 7.60-$ $7.53(\mathrm{~m}, 4 \mathrm{H}), 7.39(\mathrm{t}, J=7.6 \mathrm{~Hz}, 1 \mathrm{H}), 7.24(\mathrm{t}, J=7.6 \mathrm{~Hz}, 2 \mathrm{H}), 6.98(\mathrm{~d}$, $J=8.8 \mathrm{~Hz}, 2 \mathrm{H}), 4.36(\mathrm{~s}, 2 \mathrm{H}), 3.85(\mathrm{~s}, 3 \mathrm{H})$.

${ }^{13} \mathrm{C}\left\{{ }^{1} \mathrm{H}\right\}$ NMR $\left(100 \mathrm{MHz}, \mathrm{CDCl}_{3}\right): \delta=178.3,161.3,159.5,158.3,154.8$, $152.1,141.3,141.0,138.9,138.5,138.0,132.5,131.7,129.3,128.2(2$ $\times), 125.2,123.2,123.0,122.9,121.8,120.4,118.5,114.4(2 \times), 55.3$, 32.9 .

HRMS (ESI-TOF): $m / z[\mathrm{M}+\mathrm{H}]^{+}$calcd for $\mathrm{C}_{27} \mathrm{H}_{19} \mathrm{Br}_{2} \mathrm{~N}_{2} \mathrm{O}_{3}$ : 576.9763; found: 576.9756 .

\section{2-(6-Bromopyridin-2-yl)-3-(6-bromopyridin-2-ylmethyl)-6- (naphthalen-2-yl)chromen-4-one (4v)}

Colorless solid; yield: $453 \mathrm{mg}$ (76\%); mp $182-183^{\circ} \mathrm{C}$ (hexanes/EtOAc). ${ }^{1} \mathrm{H} \mathrm{NMR}\left(400 \mathrm{MHz}, \mathrm{CDCl}_{3}\right): \delta=8.56(\mathrm{~d}, J=2.0 \mathrm{~Hz}, 1 \mathrm{H}), 8.11(\mathrm{dd}, J=8.0$, $1.6 \mathrm{~Hz}, 1 \mathrm{H}), 8.08(\mathrm{~s}, 1 \mathrm{H}), 8.07(\mathrm{~d}, J=8.8,2.4 \mathrm{~Hz}, 1 \mathrm{H}), 7.96-7.76(\mathrm{~m}, 4$ H), $7.72(\mathrm{t}, J=7.6 \mathrm{~Hz}, 1 \mathrm{H}), 7.62(\mathrm{~d}, J=8.8 \mathrm{~Hz}, 1 \mathrm{H}), 7.57(\mathrm{dd}, J=8.0,0.8$ $\mathrm{Hz}, 1 \mathrm{H}), 7.53-7.46(\mathrm{~m}, 2 \mathrm{H}), 7.41(\mathrm{t}, J=7.6 \mathrm{~Hz}, 1 \mathrm{H}), 7.28(\mathrm{~d}, J=7.6 \mathrm{~Hz}$, $1 \mathrm{H}), 7.23(\mathrm{~d}, J=8.8 \mathrm{~Hz}, 1 \mathrm{H}), 4.38(\mathrm{~s}, 2 \mathrm{H})$.

${ }^{13} \mathrm{C}\left\{{ }^{1} \mathrm{H}\right\}$ NMR $\left(100 \mathrm{MHz}, \mathrm{CDCl}_{3}\right): \delta=178.3,161.2,158.4,155.3,152.1$, $141.4,141.1,139.0,138.5,138.3,136.5,133.6,133.0,132.8,129.4$, 128.7, 128.3, 127.6, 126.5, 126.3, 126.1, 125.3, 125.1, 124.0, 123.3, $123.0,121.9,120.5,118.7,33.0$. 
HRMS (ESI-TOF): $m / z[M+\mathrm{H}]^{+}$calcd for $\mathrm{C}_{30} \mathrm{H}_{19} \mathrm{Br}_{2} \mathrm{~N}_{2} \mathrm{O}_{2}$ : 596.9813; found: 596.9816 .

\section{6-(Biphenyl-4-yl)-2-(6-bromopyridin-2-yl)-3-(6-bromopyridin-2-} ylmethyl)chromen-4-one (4w)

Colorless solid; yield: $460 \mathrm{mg}$ (74\%); mp $212-213{ }^{\circ} \mathrm{C}$ (hexanes/EtOAc). ${ }^{1} \mathrm{H} \mathrm{NMR}\left(400 \mathrm{MHz}, \mathrm{CDCl}_{3}\right): \delta=8.47(\mathrm{~d}, J=2.4 \mathrm{~Hz}, 1 \mathrm{H}), 8.08$ (d, $J=7.6$ $\mathrm{Hz}, 1 \mathrm{H}$ ), 7.98 (dd, $J=8.8,2.4 \mathrm{~Hz}, 1 \mathrm{H}), 7.74-7.55$ (m, $9 \mathrm{H}), 7.48-7.35$ $(\mathrm{m}, 4 \mathrm{H}), 7.26(\mathrm{t}, J=7.6 \mathrm{~Hz}, 1 \mathrm{H}), 7.25(\mathrm{t}, J=8.0 \mathrm{~Hz}, 1 \mathrm{H}), 4.38(\mathrm{~s}, 2 \mathrm{H})$. ${ }^{13} \mathrm{C}\left\{{ }^{1} \mathrm{H}\right\}$ NMR $\left(100 \mathrm{MHz}, \mathrm{CDCl}_{3}\right): \delta=178.2,161.2,158.3,155.2,152.0$, 141.4, 141.1, 140.6, 140.3, 138.9, 138.5, 138.0, 137.8, 132.7, 129.3, $128.8(2 \times), 127.6(2 \times), 127.5,127.4(2 \times), 127.0(2 \times), 125.2,123.6$, 123.3, 122.9, 121.9, 120.5, 118.7, 32.9.

HRMS (ESI-TOF): $m / z[\mathrm{M}+\mathrm{H}]^{+}$calcd for $\mathrm{C}_{32} \mathrm{H}_{21} \mathrm{Br}_{2} \mathrm{~N}_{2} \mathrm{O}_{2}: 622.9970$; found: 622.9974 .

\section{2-(Pyridin-2-yl)-3-(pyridin-2-ylmethyl)chromen-4-one (4x)}

Colorless solid; yield: $220 \mathrm{mg}$ (70\%); mp $145-146{ }^{\circ} \mathrm{C}$ (hexanes/EtOAc). ${ }^{1} \mathrm{H}$ NMR $\left(400 \mathrm{MHz}, \mathrm{CDCl}_{3}\right): \delta=8.67(\mathrm{dd}, J=4.8,0.8 \mathrm{~Hz}, 1 \mathrm{H}), 8.40(\mathrm{dd}$, $J=4.8,0.8 \mathrm{~Hz}, 1 \mathrm{H}), 8.19$ (dd, $J=8.4,1.2 \mathrm{~Hz}, 1 \mathrm{H}), 7.99$ (d, $J=8.0 \mathrm{~Hz}, 1$ $\mathrm{H}), 7.78(\mathrm{dt}, J=8.0,1.6 \mathrm{~Hz}, 1 \mathrm{H}), 7.64(\mathrm{dt}, J=8.4,1.6 \mathrm{~Hz}, 1 \mathrm{H}), 7.50(\mathrm{~d}$, $J=8.4 \mathrm{~Hz}, 1 \mathrm{H}), 7.49(\mathrm{dt}, J=8.0,1.6 \mathrm{~Hz}, 1 \mathrm{H}), 7.35(\mathrm{dt}, J=8.4,1.2 \mathrm{~Hz}, 1$ $\mathrm{H}), 7.33(\mathrm{dt}, J=8.8,1.2 \mathrm{~Hz}, 1 \mathrm{H}), 7.23(\mathrm{~d}, J=8.0 \mathrm{~Hz}, 1 \mathrm{H}), 7.00(\mathrm{dd}, J=$ 8.0, $1.2 \mathrm{~Hz}, 1 \mathrm{H}), 4.37$ (s, $2 \mathrm{H})$.

${ }^{13} \mathrm{C}\left\{{ }^{1} \mathrm{H}\right\}$ NMR $\left(100 \mathrm{MHz}, \mathrm{CDCl}_{3}\right): \delta=178.4,159.9,159.8,155.9(2 \times)$, 151.6, 149.4, 148.8, 136.6, 136.0, 133.6, 125.9, 124.9, 124.5, 124.3, $122.8,120.8,120.6,118.0,33.4$.

HRMS (ESI-TOF): $m / z[\mathrm{M}+\mathrm{H}]^{+}$calcd for $\mathrm{C}_{20} \mathrm{H}_{15} \mathrm{~N}_{2} \mathrm{O}_{2}$ : 315.1134; found: 315.1129.

\section{2-(5-Fluoropyridin-2-yl)-3-(5-fluoropyridin-2-ylmethyl)- chromen-4-one (4y)}

Colorless solid; yield: $259 \mathrm{mg}$ (74\%); $\mathrm{mp} 149-150{ }^{\circ} \mathrm{C}$ (hexanes/EtOAc). $\left.{ }^{1} \mathrm{H} \mathrm{NMR} \mathrm{(400} \mathrm{MHz,} \mathrm{CDCl}_{3}\right): \delta=8.55(\mathrm{~d}, J=2.8 \mathrm{~Hz}, 1 \mathrm{H}), 8.27(\mathrm{~d}, J=2.8$ $\mathrm{Hz}, 1 \mathrm{H}), 8.21(\mathrm{dd}, J=8.0,1.6 \mathrm{~Hz}, 1 \mathrm{H}), 8.15(\mathrm{ddd}, J=8.8,4.4,0.4 \mathrm{~Hz}, 1$ $\mathrm{H}), 7.69(\mathrm{dt}, J=8.4,1.6 \mathrm{~Hz}, 1 \mathrm{H}), 7.56(\mathrm{ddd}, J=8.8,7.6,2.8 \mathrm{~Hz}, 1 \mathrm{H})$, $7.52(\mathrm{dd}, J=8.8,0.8 \mathrm{~Hz}, 1 \mathrm{H}), 7.40(\mathrm{dt}, J=8.0,1.2 \mathrm{~Hz}, 1 \mathrm{H}), 7.30(\mathrm{dt}, J=$ 8.4, $4.4 \mathrm{~Hz}, 1 \mathrm{H}), 7.26(\mathrm{dt}, J=8.8,2.8 \mathrm{~Hz}, 1 \mathrm{H}), 4.36(\mathrm{~s}, 2 \mathrm{H})$.

${ }^{13} \mathrm{C}\left\{{ }^{1} \mathrm{H}\right\}$ NMR $\left(100 \mathrm{MHz}, \mathrm{CDCl}_{3}\right): \delta=178.4,159.6(\mathrm{~d}, J=260.0 \mathrm{~Hz})$, 158.8, 158.0 (d, $J=251.7 \mathrm{~Hz}), 155.9,155.8$ (d, $J=3.8 \mathrm{~Hz}), 147.9$ (d, $J=$ $4.6 \mathrm{~Hz}), 138.0(\mathrm{~d}, J=23.5 \mathrm{~Hz}), 136.8(\mathrm{~d}, J=22.8 \mathrm{~Hz}), 133.9,126.1$, $125.8(\mathrm{~d}, J=5.3 \mathrm{~Hz}), 125.1,123.9(\mathrm{~d}, J=3.7 \mathrm{~Hz}), 123.5,123.2(\mathrm{~d}, J=$ $25.0 \mathrm{~Hz}$ ), 122.9 (d, $J=1.5 \mathrm{~Hz}), 120.7,118.0,32.6$ (d, $J=1.5 \mathrm{~Hz})$.

HRMS (ESI-TOF): $m / z[\mathrm{M}+\mathrm{H}]^{+}$calcd for $\mathrm{C}_{20} \mathrm{H}_{13} \mathrm{~F}_{2} \mathrm{~N}_{2} \mathrm{O}_{2}$ : 351.0945; found: 351.0938 .

\section{2-(6-Chloropyridin-2-yl)-3-(6-chloropyridin-2-ylmethyl)- chromen-4-one (4z)}

Colorless solid; yield: $283 \mathrm{mg}$ (74\%); mp $164-165^{\circ} \mathrm{C}$ (hexanes/EtOAc). ${ }^{1} \mathrm{H} \mathrm{NMR}\left(400 \mathrm{MHz}, \mathrm{CDCl}_{3}\right): \delta=8.15(\mathrm{dd}, J=8.0,1.6 \mathrm{~Hz}, 1 \mathrm{H}), 7.997(\mathrm{dd}$, $J=7.6,0.4 \mathrm{~Hz}, 1 \mathrm{H}), 7.77(\mathrm{t}, J=8.0 \mathrm{~Hz}, 1 \mathrm{H}), 7.63(\mathrm{dt}, J=8.8,1.6 \mathrm{~Hz}, 1$ $\mathrm{H}), 7.45(\mathrm{dd}, J=8.0,1.6 \mathrm{~Hz}, 1 \mathrm{H}), 7.43(\mathrm{~d}, J=7.6 \mathrm{~Hz}, 1 \mathrm{H}), 7.35(\mathrm{dt}, J=$ $8.0,0.8 \mathrm{~Hz}, 1 \mathrm{H}), 7.33(\mathrm{dt}, J=8.0,1.2 \mathrm{~Hz}, 1 \mathrm{H}), 7.16(\mathrm{dt}, J=7.2,0.4 \mathrm{~Hz}, 1$ $\mathrm{H}), 7.02(\mathrm{dd}, J=8.0,0.8 \mathrm{~Hz}, 1 \mathrm{H}), 4.33(\mathrm{~s}, 2 \mathrm{H})$.

${ }^{13} \mathrm{C}\left\{{ }^{1} \mathrm{H}\right\}$ NMR $\left(100 \mathrm{MHz}, \mathrm{CDCl}_{3}\right): \delta=178.0,160.6,158.2,155.6,151.5$, $150.7,150.1,139.3,138.6,133.8,125.8,125.4,125.1,122.7,122.6$, $121.3,121.2,120.3,117.9,32.7$.
HRMS (ESI-TOF): $m / z[\mathrm{M}+\mathrm{H}]^{+}$calcd for $\mathrm{C}_{20} \mathrm{H}_{13} \mathrm{Cl}_{2} \mathrm{~N}_{2} \mathrm{O}_{2}$ : 383.0354; found: 383.0348 .

Single-crystal X-ray diagram: crystal of compound $\mathbf{4 z}$ was grown by slow diffusion of EtOAc into a solution of compound $\mathbf{4 z}$ in $\mathrm{CH}_{2} \mathrm{Cl}_{2}$ to yield colorless prisms. The compound crystallizes in the triclinic crystal system, space group $P \overline{1}, a=8.0551(5) \AA, b=10.7878(6) \AA, c=$ $11.1421(6) \AA, V=815.63(8) \AA^{3}, Z=2, d_{\text {calcd }}=1.560 \mathrm{~g} / \mathrm{cm}^{3}, F(000)=392$, $2 \theta$ range $2.043-26.470^{\circ}, \mathrm{R}$ indices (all data) $R 1=0.0404, \mathrm{w} R 2=$ 0.1033 .

\section{2-(6-Methylpyridin-2-yl)-3-(6-methylpyridin-2-ylmethyl)- chromen-4-one (4aa)}

Colorless solid; yield: $274 \mathrm{mg}$ (80\%); $\mathrm{mp} 77-78{ }^{\circ} \mathrm{C}$ (hexanes/EtOAc).

${ }^{1} \mathrm{H}$ NMR $\left(400 \mathrm{MHz}, \mathrm{CDCl}_{3}\right): \delta=8.22(\mathrm{dd}, J=8.0,1.6 \mathrm{~Hz}, 1 \mathrm{H}), 7.76(\mathrm{dd}$, $J=8.0,0.4 \mathrm{~Hz}, 1 \mathrm{H}), 7.68(\mathrm{t}, J=7.6 \mathrm{~Hz}, 1 \mathrm{H}), 7.65(\mathrm{dd}, J=8.4,0.8 \mathrm{~Hz}, 1$ $\mathrm{H}), 7.51(\mathrm{~d}, J=8.4 \mathrm{~Hz}, 1 \mathrm{H}), 7.38(\mathrm{t}, J=8.0 \mathrm{~Hz}, 1 \mathrm{H}), 7.37(\mathrm{t}, J=8.0 \mathrm{~Hz}, 1$ H), $7.20(\mathrm{~d}, J=7.6 \mathrm{~Hz}, 1 \mathrm{H}), 6.96(\mathrm{~d}, J=8.0 \mathrm{~Hz}, 1 \mathrm{H}), 6.88(\mathrm{~d}, J=7.6 \mathrm{~Hz}$, $1 \mathrm{H}), 4.30$ (s, $2 \mathrm{H}), 2.49$ (s, $3 \mathrm{H}), 2.46(\mathrm{~s}, 3 \mathrm{H})$.

${ }^{13} \mathrm{C}\left\{{ }^{1} \mathrm{H}\right\}$ NMR $\left(100 \mathrm{MHz}, \mathrm{CDCl}_{3}\right): \delta=178.6,160.4,159.4,158.4,157.2$, 156.0, 150.9, 136.7, 136.4, 133.6, 126.0, 124.9, 124.2, 123.0, 121.3, $120.4,120.2,119.1,118.0,33.6,24.4,24.3$.

HRMS (ESI-TOF): $m / z[\mathrm{M}+\mathrm{H}]^{+}$calcd for $\mathrm{C}_{22} \mathrm{H}_{19} \mathrm{~N}_{2} \mathrm{O}_{2}$ : 343.1447; found: 343.1442 .

\section{2-(6-Methoxypyridin-2-yl)-3-(6-methoxypyridin-2-ylmethyl)- chromen-4-one (4ab)}

Colorless solid; yield: $292 \mathrm{mg}$ (78\%); mp 124-125 ${ }^{\circ} \mathrm{C}$ (hexanes/EtOAc). ${ }^{1} \mathrm{H} \mathrm{NMR}\left(400 \mathrm{MHz}, \mathrm{CDCl}_{3}\right): \delta=8.23(\mathrm{dd}, J=8.0,1.6 \mathrm{~Hz}, 1 \mathrm{H}), 7.71-7.62$ $(\mathrm{m}, 3 \mathrm{H}), 7.51(\mathrm{dd}, J=8.4,0.4 \mathrm{~Hz}, 1 \mathrm{H}), 7.38(\mathrm{dt}, J=8.0,0.8 \mathrm{~Hz}, 1 \mathrm{H})$, $7.36(\mathrm{t}, J=7.6 \mathrm{~Hz}, 1 \mathrm{H}), 6.80(\mathrm{dd}, J=8.0,0.8 \mathrm{~Hz}, 1 \mathrm{H}), 6.76(\mathrm{~d}, J=6.8 \mathrm{~Hz}$, $1 \mathrm{H}), 6.45(\mathrm{~d}, J=8.4 \mathrm{~Hz}, 1 \mathrm{H}), 4.53(\mathrm{~s}, 2 \mathrm{H}), 3.73(\mathrm{~s}, 3 \mathrm{H}), 3.65(\mathrm{~s}, 3 \mathrm{H})$.

${ }^{13} \mathrm{C}\left\{{ }^{1} \mathrm{H}\right\}$ NMR $\left(100 \mathrm{MHz}, \mathrm{CDCl}_{3}\right): \delta=178.6,163.2,163.1,159.2,157.7$, 155.8, 149.3, 138.8, 138.5, 133.6, 126.0, 124.8, 122.8, 120.2, 117.8, $116.9,114.7,112.6,10.3,53.4,52.9,32.6$.

HRMS (ESI-TOF): $m / z$ [M + H] $]^{+}$calcd for $\mathrm{C}_{22} \mathrm{H}_{19} \mathrm{~N}_{2} \mathrm{O}_{4}$ : 375.1345 ; found: 375.1337.

\section{3-Benzyl-2-phenylchromen-4-one (4ac)}

Colorless solid; yield: $134 \mathrm{mg}$ (43\%); mp 82-83 ${ }^{\circ} \mathrm{C}$ (hexanes/EtOAc). ${ }^{1} \mathrm{H} \mathrm{NMR}\left(400 \mathrm{MHz}, \mathrm{CDCl}_{3}\right): \delta=8.27(\mathrm{dd}, J=8.0,1.6 \mathrm{~Hz}, 1 \mathrm{H}), 7.67(\mathrm{dt}$, $J=8.4,1.6 \mathrm{~Hz}, 1 \mathrm{H}), 7.58-7.56(\mathrm{~m}, 2 \mathrm{H}), 7.52-7.46(\mathrm{~m}, 3 \mathrm{H}), 7.41(\mathrm{dt}, J=$ 8.0, 0.8 Hz, $1 \mathrm{H}), 7.25-7.21$ (m, $2 \mathrm{H}), 7.17-7.13(\mathrm{~m}, 4 \mathrm{H}), 3.98$ (s, $2 \mathrm{H})$.

${ }^{13} \mathrm{C}\left\{{ }^{1} \mathrm{H}\right\}$ NMR $\left(100 \mathrm{MHz}, \mathrm{CDCl}_{3}\right): \delta=178.2,162.9,156.2,140.1,133.5$, 133.2, 130.4, $128.6(2 \times), 128.5(2 \times), 128.4(2 \times), 128.1(2 \times), 126.1$, 125.9, 124.9, 123.0, 120.6, 117.9, 31.2.

HRMS (ESI-TOF): $m / z[\mathrm{M}+\mathrm{H}]^{+}$calcd for $\mathrm{C}_{22} \mathrm{H}_{17} \mathrm{O}_{2}: 313.1229$; found: 313.1221.

\section{2-(Naphthalen-2-yl)-3-(naphthalen-2-ylmethyl)chromen-4-one (4ad)}

Colorless solid; yield: $148 \mathrm{mg}$ (36\%); mp $144-145^{\circ} \mathrm{C}$ (hexanes/EtOAc). ${ }^{1} \mathrm{H}$ NMR $\left(400 \mathrm{MHz}, \mathrm{CDCl}_{3}\right): \delta=8.34(\mathrm{dd}, J=8.0,1.6 \mathrm{~Hz}, 1 \mathrm{H}), 8.06(\mathrm{~s}, 1$ $\mathrm{H}), 7.93(\mathrm{~d}, J=8.4 \mathrm{~Hz}, 1 \mathrm{H}), 7.91(\mathrm{~d}, J=8.8 \mathrm{~Hz}, 1 \mathrm{H}), 7.81-7.67(\mathrm{~m}, 6 \mathrm{H})$, 7.61-7.52 (m, 4 H), 7.47-7.35 (m, 4 H), 4.20 (s, 2 H). 
${ }^{13} \mathrm{C}\left\{{ }^{1} \mathrm{H}\right\}$ NMR $\left(100 \mathrm{MHz}, \mathrm{CDCl}_{3}\right): \delta=178.4,163.2,156.3,138.0,133.9$, 133.6, 132.5, 132.1, 130.4, 129.1, 128.7, 128.4, 128.0, 127.8, 127.6 (3 ×), 127.5, 127.1, 126.8, $126.2(2 \times), 125.8,125.18,125.17,125.0,123.1$, 120.8, 118.0, 31.6.

HRMS (ESI-TOF): $m / z[\mathrm{M}+\mathrm{H}]^{+}$calcd for $\mathrm{C}_{30} \mathrm{H}_{21} \mathrm{O}_{2}: 413.1542$; found: 413.1536.

\section{2-(Furan-2-yl)-3-(furan-2-ylmethyl)chromen-4-one (4af)}

Colorless solid; yield: $169 \mathrm{mg}$ (58\%); $\mathrm{mp} 111-112^{\circ} \mathrm{C}$ (hexanes/EtOAc). ${ }^{1} \mathrm{H}$ NMR $\left(400 \mathrm{MHz}, \mathrm{CDCl}_{3}\right): \delta=8.21(\mathrm{dd}, J=8.0,1.6 \mathrm{~Hz}, 1 \mathrm{H}), 7.66(\mathrm{dd}$, $J=1.6,0.4 \mathrm{~Hz}, 1 \mathrm{H}), 7.64(\mathrm{dt}, J=8.8,1.2 \mathrm{~Hz}, 1 \mathrm{H}), 7.46(\mathrm{dd}, J=8.4,0.8$ $\mathrm{Hz}, 1 \mathrm{H}), 7.37$ (dt, $J=8.0,0.8 \mathrm{~Hz}, 1 \mathrm{H}), 7.27(\mathrm{dd}, J=1.6,0.8 \mathrm{~Hz}, 1 \mathrm{H})$, $7.17(\mathrm{dd}, J=3.6,0.8 \mathrm{~Hz}, 1 \mathrm{H}), 6.60(\mathrm{dd}, J=3.6,1.6 \mathrm{~Hz}, 1 \mathrm{H}), 6.23(\mathrm{dd}, J=$ 3.2, $1.6 \mathrm{~Hz}, 1 \mathrm{H}), 6.05$ (dd, $J=3.2,0.8 \mathrm{~Hz}, 1 \mathrm{H}), 4.31(\mathrm{~s}, 2 \mathrm{H})$.

${ }^{13} \mathrm{C}\left\{{ }^{1} \mathrm{H}\right\}$ NMR $\left(100 \mathrm{MHz}, \mathrm{CDCl}_{3}\right): \delta=177.6,155.5,153.2,152.2,146.7$, $145.4,140.9,133.6,126.0,124.8,122.7,117.7,116.0,115.3,112.1$, $110.3,105.8,23.2$.

HRMS (ESI-TOF): $m / z[\mathrm{M}+\mathrm{H}]^{+}$calcd for $\mathrm{C}_{18} \mathrm{H}_{13} \mathrm{O}_{4}:$ 293.0814; found: 293.0809.

\section{2-(Thiophen-2-yl)-3-(thiophen-2-ylmethyl)chromen-4-one (4ag)} Colorless solid; yield: $194 \mathrm{mg}$ (60\%); mp $138-139{ }^{\circ} \mathrm{C}$ (hexanes/EtOAc). ${ }^{1} \mathrm{H} \mathrm{NMR}\left(400 \mathrm{MHz}, \mathrm{CDCl}_{3}\right): \delta=8.25(\mathrm{dd}, J=8.0,1.6 \mathrm{~Hz}, 1 \mathrm{H}), 7.68(\mathrm{dd}$, $J=7.2,1.6 \mathrm{~Hz}, 1 \mathrm{H}), 7.64(\mathrm{dd}, J=8.0,1.2 \mathrm{~Hz}, 1 \mathrm{H}), 7.61(\mathrm{dd}, J=8.8,0.8$ $\mathrm{Hz}, 1 \mathrm{H}), 7.50(\mathrm{dd}, J=8.0,0.8 \mathrm{~Hz}, 1 \mathrm{H}), 7.40(\mathrm{dt}, J=8.0,0.8 \mathrm{~Hz}, 1 \mathrm{H})$, 7.16 (dd, $J=4.8,4.0 \mathrm{~Hz}, 1 \mathrm{H}$ ), $6.12(\mathrm{dd}, J=4.8,1.2 \mathrm{~Hz}, 1 \mathrm{H}$ ), 6.90-6.87 $(\mathrm{m}, 2 \mathrm{H}), 4.36(\mathrm{~d}, J=0.8 \mathrm{~Hz}, 2 \mathrm{H})$.

${ }^{13} \mathrm{C}\left\{{ }^{1} \mathrm{H}\right\}$ NMR (100 MHz, $\mathrm{CDCl}_{3}$ ): $\delta=177.6,156.6,155.7,142.0,134.4$, 133.7, 130.6, 130.1, 127.9, 126.8, 126.1, 125.0, 124.7, 123.6, 122.6, $119.0,117.8,26.2$.

HRMS (ESI-TOF): $m / z[\mathrm{M}+\mathrm{H}]^{+}$calcd for $\mathrm{C}_{18} \mathrm{H}_{13} \mathrm{O}_{2} \mathrm{~S}_{2}: 325.0357$; found: 325.0352 .

Single-crystal X-ray diagram: crystal of compound 4ag was grown by slow diffusion of EtOAc into a solution of compound $\mathbf{4 a g}$ in $\mathrm{CH}_{2} \mathrm{Cl}_{2}$ to yield colorless prisms. The compound crystallizes in the monoclinic crystal system, space group $P 21 / n, a=9.1253(10) \AA, b=9.1493(10) \AA$, $c=18.386(2) \AA, V=1512.1(3) \AA^{3}, Z=4, d_{\text {calcd }}=1.425 \mathrm{~g} / \mathrm{cm}^{3}, F(000)=$ $672,2 \theta$ range $2.249-26.551^{\circ}, R$ indices (all data) $R 1=0.0339, \mathrm{w} R 2=$ 0.0734 .

\section{2-(Pyridin-3-yl)-3-(pyridin-3-ylmethyl)chromen-4-one (4ah)}

Colorless gum; yield: $85 \mathrm{mg}(27 \%)$.

${ }^{1} \mathrm{H}$ NMR $\left(400 \mathrm{MHz}, \mathrm{CDCl}_{3}\right): \delta=8.78(\mathrm{dd}, J=1.6,0.8 \mathrm{~Hz}, 1 \mathrm{H}$ ), 8.73 (dd, $J=4.8,1.6 \mathrm{~Hz}, 1 \mathrm{H}), 8.35$ (d, $J=3.6 \mathrm{~Hz}, 1 \mathrm{H}), 8.26(\mathrm{~d}, J=1.6 \mathrm{~Hz}, 1 \mathrm{H})$, $8.20(\mathrm{dd}, J=8.0,1.6 \mathrm{~Hz}, 1 \mathrm{H}), 7.79(\mathrm{dt}, J=8.0,2.4 \mathrm{~Hz}, 1 \mathrm{H}), 7.67(\mathrm{dt}, J=$ 8.4, $1.6 \mathrm{~Hz}, 1 \mathrm{H}$ ), 7.45-7.38 (m, $4 \mathrm{H}$ ), 7.10 (dd, $J=8.0,4.8 \mathrm{~Hz}, 1 \mathrm{H}$ ), 3.91 (s, $2 \mathrm{H})$.

${ }^{13} \mathrm{C}\left\{{ }^{1} \mathrm{H}\right\}$ NMR $\left(100 \mathrm{MHz}, \mathrm{CDCl}_{3}\right): \delta=177.5,160.1,156.0,151.4,149.2$, 149.0, 147.5, 135.8, 135.6, 135.0, 134.0, 128.9, 125.9, 125.3, 123.3 (2 $\times), 122.7,120.6,117.9,28.4$.

HRMS (ESI-TOF): $m / z$ [M+ H] $]^{+}$calcd for $\mathrm{C}_{20} \mathrm{H}_{15} \mathrm{~N}_{2} \mathrm{O}_{2}$ : 315.1134; found: 315.1136 .

\section{2-(Benzofuran-2-yl)-3-(benzofuran-2-ylmethyl)chromen-4-one} (4ai)

Colorless solid; yield: $251 \mathrm{mg}$ (64\%); mp $186-187^{\circ} \mathrm{C}$ (hexanes/EtOAc).
${ }^{1} \mathrm{H}$ NMR $\left(400 \mathrm{MHz}, \mathrm{CDCl}_{3}\right): \delta=8.27(\mathrm{dd}, J=8.4,1.6 \mathrm{~Hz}, 1 \mathrm{H}), 7.72(\mathrm{dt}$, $J=8.4,1.6 \mathrm{~Hz}, 1 \mathrm{H}), 7.69(\mathrm{dd}, J=8.0,0.8 \mathrm{~Hz}, 1 \mathrm{H}), 7.59(\mathrm{dd}, J=8.0,0.8$ $\mathrm{Hz}, 1 \mathrm{H}), 7.58(\mathrm{~s}, 1 \mathrm{H}), 7.56(\mathrm{dd}, J=8.4,0.8 \mathrm{~Hz}, 1 \mathrm{H}), 7.45-7.39(\mathrm{~m}, 4 \mathrm{H})$, $7.32(\mathrm{dt}, J=8.0,0.8 \mathrm{~Hz}, 1 \mathrm{H}), 7.17(\mathrm{dt}, J=8.8,1.6 \mathrm{~Hz}, 1 \mathrm{H}), 7.12(\mathrm{dt}, J=$ $8.0,1.6 \mathrm{~Hz}, 1 \mathrm{H}), 6.47$ (d, J = $1.2 \mathrm{~Hz}, 1 \mathrm{H}), 4.61(\mathrm{~d}, J=0.8 \mathrm{~Hz}, 2 \mathrm{H})$.

${ }^{13} \mathrm{C}\left\{{ }^{1} \mathrm{H}\right\}$ NMR $\left(100 \mathrm{MHz}, \mathrm{CDCl}_{3}\right): \delta=177.5,156.5,155.7,155.6,154.7$, $152.5,148.2,134.0,128.9,127.3,126.9,126.1,125.2,123.9,123.1$, $122.7,122.4,122.2,120.3,117.8,117.3,111.9,111.5,110.8,102.9$, 23.9.

HRMS (ESI-TOF): $m / z[\mathrm{M}+\mathrm{H}]^{+}$calcd for $\mathrm{C}_{26} \mathrm{H}_{17} \mathrm{O}_{4}$ : 393.1127; found: 393.1122.

\section{2-(Benzo[b]thiophen-2-yl)-3-(benzo[b]thiophen-2-yl)methyl- chromen-4-one (4aj)}

Colorless solid; yield: $267 \mathrm{mg}$ (63\%); $\mathrm{mp} 195-196^{\circ} \mathrm{C}$ (hexanes/EtOAc). ${ }^{1} \mathrm{H} \mathrm{NMR}\left(400 \mathrm{MHz}, \mathrm{CDCl}_{3}\right.$ ): $\delta=8.29$ (dd, $J=8.0,1.6 \mathrm{~Hz}, 1 \mathrm{H}$ ), 7.89 (dd, $J=8.0,2.0 \mathrm{~Hz}, 1 \mathrm{H}), 7.88(\mathrm{~s}, 1 \mathrm{H}), 7.83(\mathrm{dd}, J=8.4,1.6 \mathrm{~Hz}, 1 \mathrm{H}), 7.76(\mathrm{~d}$, $J=8.0 \mathrm{~Hz}, 1 \mathrm{H}), 7.72(\mathrm{dt}, J=8.8,2.0 \mathrm{~Hz}, 1 \mathrm{H}), 7.63(\mathrm{dd}, J=8.8,1.6 \mathrm{~Hz}, 1$ $\mathrm{H}), 7.56(\mathrm{~d}, J=8.0 \mathrm{~Hz}, 1 \mathrm{H}), 7.46-7.39(\mathrm{~m}, 3 \mathrm{H}), 7.29(\mathrm{dt}, J=8.4,1.2 \mathrm{~Hz}$, $1 \mathrm{H}), 7.25(\mathrm{dt}, J=8.4,1.2 \mathrm{~Hz}, 1 \mathrm{H}), 7.12(\mathrm{~s}, 1 \mathrm{H}), 4.49(\mathrm{~d}, J=0.8 \mathrm{~Hz}, 2 \mathrm{H})$. ${ }^{13} \mathrm{C}\left\{{ }^{1} \mathrm{H}\right\}$ NMR $\left(100 \mathrm{MHz}, \mathrm{CDCl}_{3}\right): \delta=177.5,156.9,155.8,143.1,140.8$, $140.1,139.6,138.9,134.0,133.9,127.8,126.3,126.2,125.3,125.0(2$ $\times), 124.1,123.6,123.0,122.6,122.2,122.1,121.3,119.7,117.9,27.2$. HRMS (ESI-TOF): $m / z[\mathrm{M}+\mathrm{H}]^{+}$calcd for $\mathrm{C}_{26} \mathrm{H}_{17} \mathrm{O}_{2} \mathrm{~S}_{2}: 425.0670$; found: 425.0678 .

\section{1-(2-Hydroxyphenyl)-3-(1-methyl-1H-indol-3-yl)prop-2-en-1-one} (4ak-1)

Colorless solid; yield: $42 \mathrm{mg}$ (15\%); $\mathrm{mp} 208-209{ }^{\circ} \mathrm{C}$ (hexanes/EtOAc). ${ }^{1} \mathrm{H}$ NMR $\left(400 \mathrm{MHz}, \mathrm{CDCl}_{3}\right): \delta=13.30(\mathrm{~s}, 1 \mathrm{H}), 8.19(\mathrm{~d}, J=15.2 \mathrm{~Hz}, 1 \mathrm{H})$, 8.03-8.00 (m, $1 \mathrm{H}), 7.96(\mathrm{dd}, J=8.4,1.6 \mathrm{~Hz}, 1 \mathrm{H}), 7.65(\mathrm{~d}, J=15.2 \mathrm{~Hz}, 1$ H), $7.50(\mathrm{~s}, 1 \mathrm{H}), 7.48(\mathrm{dt}, J=7.6,2.0 \mathrm{~Hz}, 1 \mathrm{H}), 7.41-7.33(\mathrm{~m}, 3 \mathrm{H}), 7.03$ (dd, $J=8.4,0.8 \mathrm{~Hz}, 1 \mathrm{H}), 6.96(\mathrm{dt}, J=8.0,1.2 \mathrm{~Hz}, 1 \mathrm{H}), 3.85(\mathrm{~s}, 3 \mathrm{H})$.

${ }^{13} \mathrm{C}\left\{{ }^{1} \mathrm{H}\right\}$ NMR $\left(100 \mathrm{MHz}, \mathrm{CDCl}_{3}\right): \delta=193.6,163.5,139.4,138.4,135.6$, $135.4,129.3,126.1,123.4,121.9,120.8,120.3,118.6,115.5,114.5$, 113.1, 110.3, 33.4.

HRMS (ESI-TOF): $m / z[\mathrm{M}+\mathrm{H}]^{+}$calcd for $\mathrm{C}_{18} \mathrm{H}_{16} \mathrm{NO}_{2}: 278.1181$; found: 278.1188 .

2-(Quinolin-2-yl)-3-(quinolin-2-ylmethyl)chromen-4-one (4al) Colorless solid; yield: $145 \mathrm{mg}$ (35\%); mp $122-123^{\circ} \mathrm{C}$ (hexanes/EtOAc). ${ }^{1} \mathrm{H} \mathrm{NMR}\left(400 \mathrm{MHz}, \mathrm{CDCl}_{3}\right): \delta=8.27(\mathrm{dd}, J=8.0,1.6 \mathrm{~Hz}, 1 \mathrm{H}), 8.25(\mathrm{~d}, J=$ $9.2 \mathrm{~Hz}, 1 \mathrm{H}), 8.10(\mathrm{~d}, J=8.4 \mathrm{~Hz}, 1 \mathrm{H}), 7.98(\mathrm{~d}, J=8.4 \mathrm{~Hz}, 1 \mathrm{H}), 7.92(\mathrm{dd}$, $J=8.4,3.2 \mathrm{~Hz}, 1 \mathrm{H}), 7.80(\mathrm{dd}, J=8.0,0.8 \mathrm{~Hz}, 1 \mathrm{H}), 7.71-7.53(\mathrm{~m}, 7 \mathrm{H})$, $7.46(\mathrm{~d}, J=8.8 \mathrm{~Hz}, 1 \mathrm{H}), 7.43-7.38(\mathrm{~m}, 2 \mathrm{H}), 4.71(\mathrm{~s}, 2 \mathrm{H})$.

${ }^{13} \mathrm{C}\left\{{ }^{1} \mathrm{H}\right\}$ NMR $\left(100 \mathrm{MHz}, \mathrm{CDCl}_{3}\right): \delta=178.6,160.7,160.1,156.0,151.5$, $147.7,147.3,136.7,135.9,133.7,130.0,129.9,128.9,128.8,127.9$, 127.8, 127.4, 127.3, 126.7, 126.1, 125.4, 125.1, 123.0, 121.2, 121.0, 120.8, 118.0, 34.3.

HRMS (ESI-TOF): $m / z$ [M + H] $]^{+}$calcd for $\mathrm{C}_{28} \mathrm{H}_{19} \mathrm{~N}_{2} \mathrm{O}_{2}$ : 415.1447; found: 415.1446 .

2-[6-(3,4-Dimethoxyphenyl)pyridin-2-yl]-3-[6-(3,4-dimethoxyphenyl)pyridin-2-ylmethyl]chromen-4-one (4am)

3,4-Dimethoxyphenylboronic acid (180 mg, $1.0 \mathrm{mmol}$ ), $\mathrm{Pd}(\mathrm{OAc})_{2}$ (45 mg, $20 \mathrm{~mol} \%$ ), $\mathrm{PPh}_{3}$ (130 mg, $0.5 \mathrm{mmol}$ ), and $\mathrm{Na}_{2} \mathrm{CO}_{3}(53 \mathrm{mg}, 0.5$ $\mathrm{mmol}$ ) were added stepwise to a solution of $4 \mathbf{a}(235 \mathrm{mg}, 0.5 \mathrm{mmol})$ in EtOH $(15 \mathrm{~mL})$ at $25^{\circ} \mathrm{C}$. The mixture was stirred at reflux for $8 \mathrm{~h}$ (TLC 
monitoring). The mixture was cooled to $25^{\circ} \mathrm{C}$, concentrated, and extracted with EtOAc $(3 \times 15 \mathrm{~mL})$. The combined organic layers were washed with brine, dried, filtered, and evaporated to afford the crude product under reduced pressure. Purification on silica gel (hexanes/EtOAc 8:1-2:1) afforded 4am as a colorless gum; yield: $220 \mathrm{mg}$ (75\%).

${ }^{1} \mathrm{H}$ NMR $\left(400 \mathrm{MHz}, \mathrm{CDCl}_{3}\right): \delta=8.24(\mathrm{dd}, J=8.0,1.6 \mathrm{~Hz}, 1 \mathrm{H}), 7.93(\mathrm{dd}$, $J=7.6,0.8 \mathrm{~Hz}, 1 \mathrm{H}), 7.85(\mathrm{~d}, J=8.0 \mathrm{~Hz}, 1 \mathrm{H}), 7.76(\mathrm{dd}, J=8.0,0.8 \mathrm{~Hz}, 1$ $\mathrm{H}), 7.70(\mathrm{dt}, J=8.4,1.6 \mathrm{~Hz}, 1 \mathrm{H}), 7.56-7.52(\mathrm{~m}, 4 \mathrm{H}), 7.45-7.37(\mathrm{~m}, 4$ H), $7.17(\mathrm{~d}, J=7.6 \mathrm{~Hz}, 1 \mathrm{H}), 6.83(\mathrm{~d}, J=8.4 \mathrm{~Hz}, 1 \mathrm{H}), 6.79(\mathrm{~d}, J=8.8 \mathrm{~Hz}$, $1 \mathrm{H}), 4.70$ (s, $2 \mathrm{H}), 3.87$ (s, $3 \mathrm{H}), 3.84(\mathrm{~s}, 3 \mathrm{H}), 3.71(\mathrm{~s}, 3 \mathrm{H}), 3.70(\mathrm{~s}, 3 \mathrm{H})$.

${ }^{13} \mathrm{C}\left\{{ }^{1} \mathrm{H}\right\}$ NMR $\left(100 \mathrm{MHz}, \mathrm{CDCl}_{3}\right): \delta=178.7,159.7,159.6,156.6,156.0$, 155.8, 151.7, 150.2, 149.5, 149.1, 148.9, 137.3, 136.8, 133.7, 132.6, 131.2, 126.0, 124.9, 122.9, 121.7, 121.0, 120.5 (2×), 119.6, 119.0, $117.9,116.8,111.0,110.7,110.0,109.9,55.84,55.81,55.7,55.5,33.5$.

HRMS (ESI-TOF): $m / z[\mathrm{M}+\mathrm{H}]^{+}$calcd for $\mathrm{C}_{36} \mathrm{H}_{31} \mathrm{~N}_{2} \mathrm{O}_{6}$ : 587.2182; found: 587.2188.

\section{2-(6-Bromopyridin-2-yl)-3-[6-(3,4-dimethoxyphenyl)pyridin-2- ylmethyl]chromen-4-one (4am-1)}

Colorless solid; yield: $26 \mathrm{mg}$ (10\%); mp $188-190{ }^{\circ} \mathrm{C}$ (hexanes/EtOAc). ${ }^{1} \mathrm{H} \mathrm{NMR}\left(400 \mathrm{MHz}, \mathrm{CDCl}_{3}\right): \delta=8.20(\mathrm{dd}, J=8.0,1.6 \mathrm{~Hz}, 1 \mathrm{H}), 7.89(\mathrm{dd}$, $J=7.6,1.2 \mathrm{~Hz}, 1 \mathrm{H}), 7.84(\mathrm{~d}, J=7.6 \mathrm{~Hz}, 1 \mathrm{H}), 7.73(\mathrm{dd}, J=7.6,1.2 \mathrm{~Hz}, 1$ H), $7.67(\mathrm{dt}, J=8.4,1.2 \mathrm{~Hz}, 1 \mathrm{H}), 7.52(\mathrm{~d}, J=8.4 \mathrm{~Hz}, 1 \mathrm{H}), 7.42(\mathrm{~d}, J=2.0$ $\mathrm{Hz}, 1 \mathrm{H}), 7.38(\mathrm{t}, J=7.6 \mathrm{~Hz}, 1 \mathrm{H}), 7.31(\mathrm{t}, J=7.6 \mathrm{~Hz}, 1 \mathrm{H}), 7.26(\mathrm{dd}, J=$ 8.4, 2.0 Hz, $1 \mathrm{H}), 7.20$ (d, J = 7.6 Hz, $1 \mathrm{H}), 7.14(\mathrm{~d}, J=7.6 \mathrm{~Hz}, 1 \mathrm{H}), 6.85$ (d, $J=8.4 \mathrm{~Hz}, 1 \mathrm{H}), 4.60$ (s, $2 \mathrm{H}), 3.89(\mathrm{~s}, 3 \mathrm{H}), 3.78(\mathrm{~s}, 3 \mathrm{H})$.

${ }^{13} \mathrm{C}\left\{{ }^{1} \mathrm{H}\right\}$ NMR $\left(100 \mathrm{MHz}, \mathrm{CDCl}_{3}\right): \delta=178.4,161.8,159.9,156.5,155.8$, $151.2,150.2,149.0,141.0,138.4,137.4,133.8,131.0,125.8,125.0(2$ ×), 122.7, 121.6, 120.9, 120.7, 119.7, 119.5, 117.9, 111.0, 109.7, 55.8, 55.7, 33.3.

HRMS (ESI-TOF): $m / z[\mathrm{M}+\mathrm{H}]^{+}$calcd for $\mathrm{C}_{28} \mathrm{H}_{22} \mathrm{BrN}_{2} \mathrm{O}_{4}: 529.0763$; found: 529.0769 .

\section{2-(2-Allyl-3,4-dimethoxyphenyl)chromen-4-one (4an)}

$\mathrm{NaOH}$ (80 mg, $2.0 \mathrm{mmol}$ ) was added to a solution of $\mathbf{2 a}$ (136 mg, 1.0 $\mathrm{mmol})$ in $\mathrm{MeOH}(30 \mathrm{~mL})$ at $25{ }^{\circ} \mathrm{C}$ and the mixture was stirred at $25^{\circ} \mathrm{C}$ for $10 \mathrm{~min}$. Compound 3q (412 mg, $2.0 \mathrm{mmol}$ ) was added to the mixture at $25^{\circ} \mathrm{C}$ and then the mixture was stirred at $50{ }^{\circ} \mathrm{C}$ for $10 \mathrm{~h}$. The mixture was cooled to $25{ }^{\circ} \mathrm{C}$ and the solvent was concentrated. The residue was diluted with water $(10 \mathrm{~mL})$ and the mixture was extracted with $\mathrm{CH}_{2} \mathrm{Cl}_{2}(3 \times 20 \mathrm{~mL})$. The combined organic layers were washed with brine, dried, filtered and evaporated to afford the crude product under reduced pressure. Purification (silica gel, hexanes/EtOAc 10:14:1) afforded 4an as a colorless solid; yield: $129 \mathrm{mg}$ (40\%); mp 208$209{ }^{\circ} \mathrm{C}$ (hexanes/EtOAc).

${ }^{1} \mathrm{H}$ NMR $\left(400 \mathrm{MHz}, \mathrm{CDCl}_{3}\right): \delta=8.28(\mathrm{dd}, J=8.0,1.6 \mathrm{~Hz}, 1 \mathrm{H}), 7.69(\mathrm{dt}$, $J=8.4,1.6 \mathrm{~Hz}, 1 \mathrm{H}$ ), 7.49 (dd, $J=8.4,0.8 \mathrm{~Hz}, 1 \mathrm{H}), 7.43$ (dt, $J=8.0,0.8$ $\mathrm{Hz}, 1 \mathrm{H}), 7.36(\mathrm{~d}, J=8.4 \mathrm{~Hz}, 1 \mathrm{H}), 6.95(\mathrm{~d}, J=8.4 \mathrm{~Hz}, 1 \mathrm{H}), 6.42(\mathrm{~s}, 1 \mathrm{H})$, 5.95-5.85 (m, 1 H), 4.91-4.82 (m, 2 H), $3.94(\mathrm{~s}, 3 \mathrm{H}), 3.87$ (s, $3 \mathrm{H}), 3.51$ (dt, $J=6.4,1.6 \mathrm{~Hz}, 2 \mathrm{H})$.

${ }^{13} \mathrm{C}\left\{{ }^{1} \mathrm{H}\right\}$ NMR $\left(100 \mathrm{MHz}, \mathrm{CDCl}_{3}\right): \delta=173.2,155.6,154.4,147.8,147.7$, $138.4,136.6,133.9,133.5,126.5,125.5,124.5,123.0,121.2,118.4$, 115.2, 110.2, 60.8, 55.8, 31.8.

HRMS (ESI-TOF): $m / z[\mathrm{M}+\mathrm{H}]^{+}$calcd for $\mathrm{C}_{20} \mathrm{H}_{19} \mathrm{O}_{4}$ : 323.1283; found: 323.1288 .

\section{Funding Information}

The authors would like to thank the Ministry of Science and Technology, Taiwan for its financial support (MOST 106-2628-M-037-001MY3).

\section{Supporting Information}

Supporting information for this article is available online at https://doi.org/10.1055/s-0039-1690760. Included are scanned photocopies of NMR spectral data for all compounds and X-ray analysis data of $4 a-4 d, 4 g, 4 t, 4 z$ and $4 a g$.

\section{References}

(1) For reviews, see: (a) Kosmider, B.; Osiecka, R. Drug Dev. Res. 2004, 63, 200. (b) Teillet, F.; Boumendjel, A.; Boutonnat, J.; Ronot, X. Med. Res. Rev. 2008, 28, 715. (c) Talhi, O.; Silva, A. M. S. Curr. Org. Chem. 2012, 16, 859. (d) Kumazawa, Y.; Takimoto, H.; Matsumoto, T.; Kawaguchi, K. Curr. Pharm. Res. 2014, 20, 857. (e) Ibrahim, M. A.; Ali, T. E.; Alnamer, Y. A.; Gabr, Y. A. ARKIVOC 2010, (i), 98. (f) Gaspar, A.; Matos, M. J.; Garrido, J.; Uriarte, E.; Borges, F. Chem. Rev. 2014, 114, 4960. (g) Plaskon, A. S.; Grygorenko, O. O.; Ryabukhin, S. V. Tetrahedron 2012, 68, 2743. (h) Li, N.-G.; Shi, Z.-H.; Tang, Y.-P.; Ma, H.-Y.; Yang, J.-P.; Li, B.-Q.; Wang, Z.-J.; Song, S.-L.; Duan, J. A. J. Heterocycl. Chem. 2010, 47, 785. (i) Snatos, C. M. M.; Silva, A. M. S. Eur. J. Org. Chem. 2017, 2017, 3115.

(2) Selected recent examples on biological activities of flavones, see: (a) Badavath, V. N.; Nath, C.; Ganta, N. M.; Ucar, G.; Sinha, B. N.; Jayaprakash, V. Chin. Chem. Lett. 2017, 28, 1528. (b) Charvin, D.; Pomel, V.; Ortiz, M.; Frauli, M.; Scheffler, S.; Steinberg, E.; Baron, L.; Deshons, L.; Rudigier, R.; Thiarc, D.; Morice, C.; Manteau, B.; Mayer, S.; Graham, D.; Giethlen, B.; Brugger, N.; Hédou, G.; Conquet, F.; Schann, S. J. Med. Chem. 2017, 60, 8515. For a recent review, see: (c) Reis, J.; Gaspar, A.; Milhazes, N.; Borges, F. J. Med. Chem. 2017, 60, 7941.

(3) Selected examples on biological activities of homoisoflavanones, see: (a) Basavarajappa, H. D.; Lee, B.; Lee, H.; Sulaiman, R. S.; An, H.; Magaña, C.; Shadmand, M.; Vayl, A.; Rajashekhar, G.; Kim, E. Y.; Suh, Y.-G.; Lee, K.; Seo, S.-Y.; Corson, T. W. J. Med. Chem. 2015, 58, 5015. (b) Zhang, H.; Yang, F.; Qi, J.; Song, X.-C.; Hu, Z.-F.; Zhu, D.-N.; Yu, B.-Y. J. Nat. Prod. 2010, 73, 548.

(4) Shen, C.; Li, W.; Yin, H.; Spannenberg, A.; Skrydstrup, T.; Wu, X.F. Adv. Synth. Catal. 2016, A leading example on synthesis of flavones, see: 358,46 ; and references cited therein.

(5) Selected examples on synthesis of homoisoflavanones and their derivatives, see: (a) Hu, H.; Chen, X.; Sun, K.; Wang, J.; Liu, Y.; Liu, H.; Fan, L.; Yu, B.; Sun, Y.; Qu, L.; Zhao, Y. Org. Lett. 2018, 20 , 6157. (b) Lee, B.; Basavarajappa, H. D.; Sulaiman, R. S.; Fei, X.; Seo, S.-Y.; Corson, T. W. Org. Biomol. Chem. 2014, 12, 7673. (c) Poisson, T.; Gembus, V.; Dalla, V.; Oudeyer, S.; Levacher, V. J. Org. Chem. 2010, 75, 7704. (d) Mrug, G. P.; Myshko, N. V.; Bondarenko, S. P.; Sviripa, V. M.; Frasinyuk, M. S. J. Org. Chem. 2019, 84, 7138 .

(6) For biological activities of 2-aryl-3-(imidazolylmethyl)chromones, see: (a) Recanatini, M.; Bisi, A.; Cavalli, A.; Belluti, F.; Gobbi, S.; Rampa, A.; Valenti, P.; Palzer, M.; Palusczak, A.; Hartmann, R. W. J. Med. Chem. 2001, 44, 672. (b) Gobbi, S.; Cavalli, A.; Rampa, A.; Belluti, F.; Piazzi, L.; Paluszcak, A.; 
Hartmann, R. W.; Recanatini, M.; Bisi, A. J. Med. Chem. 2006, 49, 4777. (c) Gobbi, S.; Hu, Q.; Zimmer, C.; Engel, M.; Belluti, F.; Rampa, A.; Harmann, R. W.; Bisi, A. J. Med. Chem. 2016, 59, 2468.

(7) For synthesis of 2-aryl-3-(arylmethyl)chromones, see: (a) Raja, G. C. E.; Ryu, J. Y.; Lee, J.; Lee, S. Org. Lett. 2017, 19, 6606. (b) Zhao, X.; Zhou, J.; Lin, S.; Jin, X.; Liu, R. C-H. Org. Lett. 2017, $19,976$.

(8) (a) Chang, M.-Y.; Wu, M.-H. Tetrahedron Lett. 2012, 53, 3173.

(b) Chang, M.-Y.; Wu, M.-H.; Tai, H.-Y. Org. Lett. 2012, 14, 3936.

(c) Chang, M.-Y.; Wu, M.-H. Tetrahedron 2012, 68, 9616.

(9) (a) Hofmann, E.; Webster, J.; Do, T.; Kline, R.; Snider, L.; Hauser, Q.; Higginbottom, G.; Campbell, A.; Ma, L.; Paula, S. Bioorg. Med. Chem. 2016, 24, 578. (b) Stoyanov, E. V.; Champavier, Y.; Simon,
A.; Basly, J.-P. Bioorg. Med. Chem. Lett. 2002, 12, 2685. (c) Guo, G.; Wan, S.; Si, X.; Jiang, Q.; Jia, Y.; Yang, L.; Zhou, W. Org. Lett. 2017, 19, 5026.

(10) (a) Colombe, J. R.; Bernhardt, S.; Stathakis, C.; Buchwald, S. L.; Knochel, P. Org. Lett. 2013, 15, 5754. (b) Štefko, M.; Slavětínská, L.; Klepetářová, B.; Hocek, M.J. Org. Chem. 2010, 75, 442. (c) Wu, G. G.; Wong, Y. S.; Poirier, M. Org. Lett. 1999, 1, 745.

(11) CCDC 1896972 (4a), 1896973 (4b), 1896974 (4c), 1896975 (4d), 1896977 (4g), 1896976 (4t), 1896978 (4z), and 1896979 (4ag) contain the supplementary crystallographic data for this paper. The data can be obtained free of charge from The Cambridge Crystallographic Data Centre via www.ccdc.cam.ac.uk/getstructures.

(12) Wachter-Jurcsak, N.; Radu, C.; Redin, K. Tetrahedron Lett. 1998, 39, 3903. 